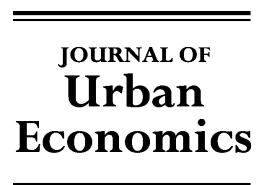

www.elsevier.com/locate/jue

\title{
Duality in comparative statics in rental housing markets with indivisibilities
}

\author{
Mamoru Kaneko*, Tamon Ito, Yu-ichi Osawa \\ Institute of Policy and Planning Sciences, University of Tsukuba, Ibaraki 305-8573, Japan
}

Received 25 January 2005; revised 26 September 2005

Available online 10 November 2005

\begin{abstract}
We present certain duality results on comparative statics on competitive rent vectors in the rental housing market model. In the model, apartments as indivisible goods are classified into a finite number of categories, and are traded for one composite commodity. Our concern is about certain general properties of the behavior of rents with parameter changes. In particular, the rent changes are intimately related to the boundary income changes of the categories of apartments. Both changes are endogenously determined in equilibrium. We will show that these changes exhibit nice dual structures. We will also apply our model and comparative statics to a rental housing market in the Tokyo metropolitan area.
\end{abstract}

(C) 2005 Elsevier Inc. All rights reserved.

JEL classification: R31; R30; D58

Keywords: Rental housing; Apartment; Indivisible goods; Competitive equilibrium; Rent equation; Comparative statics; Duality

\section{Introduction}

This paper aims to present certain duality results on comparative statics on competitive rent vectors in the rental housing market model. In the model, apartments as indivisible goods are classified into a finite number of categories by some attributes such as sizes, commuting times and housing regions, and they are traded for one composite commodity. By comparative statics, we mean a study of the behavior of rents when some parameters of the market change. Our concern

\footnotetext{
* Corresponding author.

E-mail addresses: kaneko@shako.sk.tsukuba.ac.jp (M. Kaneko), taito@shako.sk.tsukuba.ac.jp (T. Ito), osawa50@shako.sk.tsukuba.ac.jp (Y. Osawa).
} 
is not a particular form of "cause-effect" comparative statics, but it is about certain general properties of the behavior of rents with parameter changes. We focus on the boundary income, i.e., the lowest income, in each category. The changes in rent differences are intimately related to the changes in boundary income differences. Both changes are endogenously determined in equilibrium. We will show that these changes exhibit nice dual structures.

This introduction will first give brief explanations of the relation of our housing model to some urban economics literatures as well as to the literature of markets with indivisible goods, and then will explain our comparative statics.

Our model is a partial equilibrium model in that all goods other than housing are treated as one composite commodity. But it admits commodity differentiations in apartments as well as income effects on households' behavior. Our model is a variant of the bid-offer model in the standard urban economics literature from Alonso [1] and Muth [18] (see Fujita [7] for a recent textbook), where housing sizes are treated as continuous variables. A salient difference of our model from this literature is that each housing unit is indivisible, and also, the number of apartment units is finite.

In another literature in urban economics such as Sweeney [22], Braid [5] and van Lierop [24], apartments are treated as indivisible. However, the housing quality is expressed as a continuous variable. The mathematical methodology used in these urban economics literatures is dominantly calculus and analysis. Our approach differs from those literatures in that our housing model is of finite nature, except the composite commodity. This requires a different mathematical method, which is of combinatorics nature. We show that this finite nature is able to capture the housing market structure well.

The mathematical model of this paper also belongs to the other tradition from BöhmBawerk [25], Neumann-Morgenstern [26] and Shapley-Shubik [21] (see Laan et al. [16] and its references for recent papers). Nevertheless, this tradition has had a theoretical focus on the existence of competitive equilibria and the nonemptiness of the core, etc. Only Kaneko [15] and Gerber [9] tried to apply this approach to urban housing market problems. The present paper is a further development of [15].

Our approach has both advantages and disadvantages to the standard bid-offer approach. It is a disadvantage, caused by the indivisibility assumption, that the finite number of housing sizes are already fixed, while a household can choose freely a size or quality in the standard approach. This disadvantage restricts our scope to housing markets where houses are dwellings, i.e., they are already built and their sizes are fixed. With the cost of this disadvantage, our restriction can be regarded as advantageous in a quite few respects over the standard approach. For example, it will be clear by giving certain numerical examples that our model is flexible enough to capture rental housing markets well. The main theme of this paper, i.e., comparative statics, is also an advantage.

We should emphasize that our approach is short-run in the sense that we treat housing units as already produced and provided to the market. It may be found in the numerical examples given in Sections 3 and 7 that our approach is even regarded as snapshot in that the housing market at a point of time is studied. This is in contrast with the long-run approach such as in Braid [6]. ${ }^{1}$

Let us give a brief description of our housing market model. Our model has various differentiated apartments and one composite commodity. Section 2 will start with a general formulation, while referring to some preceding works on the existence of a competitive equilibrium. There the

\footnotetext{
1 Cf., also Sweeney [23], Ohls [20] and Arnott-Braid [2].
} 
households are allowed to have different utility functions. Then we assume that every household has the same utility function, and make a few specific assumptions on the utility function, e.g., the normality of apartment qualities. Although the households have the same utility function, they may have different incomes, which cause their different behaviors of housing consumption.

With the above specifications, we focus on a certain system of equations, which we call the rent equation. Its solution is a differential rent vector. It can be regarded as representing competitive rents in our model: Section 2.3 gives sufficient conditions for the equivalence between a differential rent vector and a competitive rent vector. Its basic logic is the same as Ricardo's [19] and Alonso's [1] argument of differential rents. Technically, it is also corresponding to the envelope curve of the bid-offer curves in the bid-offer model (e.g., Fujita [7, Section 3.6] Braid [5] and Gerber [9]). In our comparative study, we will consider a differential rent vector as a proxy of a competitive rent vector.

We should mention the two key concepts to determine the rent equation:

(i) the marginal category, $f$, of apartments;

(ii) the boundary household, $G(k)$, of each category, $k$, of apartments.

Recall that the apartments are classified into a finite number of categories. The marginal category corresponds to the marginal land in the Ricardian argument. We put richer households into better categories of apartments, and then the boundary household in each category has the lowest income in the category. In fact, this is a result in competitive equilibrium. Thus, this boundary household is endogenously determined. The income level of the boundary household in a category is called the boundary income of the category.

In Section 2.3, the rent equation will be formulated, using the marginal category and boundary households, and it states that the boundary household of each category is indifferent between an apartment in his category and one in the one-rank lower category.

Now we are in a state to describe our comparative statics results. As mentioned in the beginning of this introduction, our concern is not a particular cause-effect form of comparative statics; instead, it is the consideration of certain general properties of the behavior of a differential rent vector with changes in the boundary incomes. We will focus on the boundary income of each category $k$. Now, let $I_{G(k)}$ be the boundary income of category $k$, and $r_{k}$ the rent in category $k$. Suppose that $I_{G(k)}$ and $r_{k}$ change into new ones $\hat{I}_{\widehat{G}(k)}$ and $\hat{r}_{k}$. The rent differences $\hat{r}_{k}-r_{k}$ are intimately related to the boundary income differences $\hat{I}_{\widehat{G}(k)}-I_{G(k)}$. Our comparative statics results will be stated in terms of these differences. Note that $\hat{r}_{k}-r_{k}$ and $\hat{I}_{\widehat{G}(k)}-I_{G(k)}$ are endogenously determined in equilibrium.

The incomes of households $1, \ldots, m$ are ordered in the descending way, i.e., $I_{1} \geqslant I_{2} \geqslant \ldots$ $\geqslant I_{m}>0$. Two cases of changes in the boundary incomes are considered:

(1) $\hat{I}_{\widehat{G}(1)}-I_{G(1)} \geqslant \hat{I}_{\widehat{G}(2)}-I_{G(2)} \geqslant \cdots \geqslant \hat{I}_{\widehat{G}(f-1)}-I_{G(f-1)}$;

(2) $\hat{I}_{\widehat{G}(1)}-I_{G(1)} \leqslant \hat{I}_{\widehat{G}(2)}-I_{G(2)} \leqslant \cdots \leqslant \hat{I}_{\widehat{G}(f-1)}-I_{G(f-1)}$.

Recall that $f$ is the marginal category of apartments. In case (1), the boundary income difference is larger for a better category of apartments; and the opposite in (2). In these two cases, the comparative statics results between the income and rent differences exhibit a dual structure. By a dual structure, we mean that a comparative statics proposition for (2) is obtained from a proposition for (1) by replacing the inequalities with the opposite inequalities, and vice versa. 
In Sections 5 and 6, we will give various theorems on comparative statics, which have duality properties.

In Sections 3 and 7, we will exemplify the model and some theorems in a rental housing market of the residential area along the Chuo railway line in Tokyo. In those examples, we consider not only numerical examples but compare the computational results of rents with the data form the Chuo railway line. We consider how the numerical calculation fits to the data. Using those numerical examples, we illustrate our comparative statics results.

Some results in this paper are restatements already found in a more specific model in Kaneko [15], though we follow his specification in the numerical example. The present paper gives a better discourse of the rental housing market with indivisibilities than [15].

The paper consists of eight sections including this introduction. In Section 2, we will provide a basic framework and state basic results. In Section 3, we will start an illustration of a rental housing market example in Tokyo. In Section 4, we will formulate comparative statics and mention one basic theorem for comparative statics. In Sections 5 and 6, we will give various theorems on the dual treatments of the rent differences. In Section 7, we will give more explanation the numerical example given in Section 3 and will illustrate some comparative statics results. In Section 8 , we will conclude this paper with some remarks on future possible researches as well as generalizations of certain results obtained in this paper.

\section{Rental housing markets}

We begin this section with a general formulation of a rental housing market model as well as that of competitive equilibrium. In our comparative study, we will not directly use competitive equilibrium, but will adopt a differential rent vector and the rent equation. Nevertheless, their definitions need a precise description of a housing market model as well as that of competitive equilibrium. We will give certain sufficient conditions for a competitive rent vector to be a differential rent vector.

\subsection{General formulation}

The rental housing market model we consider is denoted by $(M, N)$. The first symbol $M$ is the set of households, $\{1, \ldots, m\}$, each of whom looks for one unit of an apartment, and the second symbol $N$ is the set of landlords, $\left\{1^{\prime}, \ldots, n^{\prime}\right\}$, each of whom supplies some units of apartments to the market. The apartments are classified into $T$ categories, $1, \ldots, T$. These $T$ categories of apartments are interpreted as potentially supplied. If no confusion is expected, we would use the word "apartment" for either one unit or a category of apartments.

Each household $i \in M$ wants to rent at most one apartment (for a given period of time). He chooses a consumption bundle $\left(x_{i}, c\right)$ from the consumption set $X:=\left\{\mathbf{0}, \mathbf{e}^{1}, \ldots, \mathbf{e}^{T}\right\} \times \mathbf{R}_{+}$, where each $\mathbf{e}^{k}$ is the unit $T$-vector with its $k$ th component 1 and $\mathbf{R}_{+}$is the set of nonnegative real numbers. The inclusion of vector $\mathbf{0}$ means that household $i$ may not rent an apartment in this market. We denote the zero vector $\mathbf{0}$ by $\mathbf{e}^{0}$. A typical element $\left(\mathbf{e}^{k}, c\right)$ means that household $i$ rents one unit of the $k$ th category of apartments and enjoys the consumption $c=I_{i}-p_{k}$ of the composite commodity after paying the rent $p_{k}$ for the one unit $\mathrm{e}^{k}$ from his income $I_{i}>0$.

Each household $i \in M$ has the initial endowment $\left(\mathbf{e}^{0}, I_{i}\right)$ with $I_{i}>0$. That is, household $i$ with income $I_{i}$ looks for an apartment. Also, each household $i$ has a utility function $u_{i}: X \rightarrow R$. First, we make the following assumption: 
Assumption A (Continuity and Monotonicity). For each $i \in M$ and $x \in\left\{\mathbf{e}^{0}, \mathbf{e}^{1}, \ldots, \mathbf{e}^{T}\right\}, u_{i}\left(x_{i}, c\right)$ is a continuous and strictly monotone function of $c$, and $u_{i}\left(\mathbf{e}^{0}, I_{i}\right)>u_{i}\left(\mathbf{e}^{k}, 0\right)$ for $k=1, \ldots, T$.

The last inequality, $u_{i}\left(\mathbf{e}^{0}, I_{i}\right)>u_{i}\left(\mathbf{e}^{k}, 0\right)$, means that going out of the market is preferred to renting an apartment with no consumption. This is just a boundary condition. Here we do not specify the urban structure of a housing market, and will use the utility function $u_{i}$, which will later be assumed to satisfy more assumptions. Nevertheless, the utility function $u_{i}$ is rather regarded as derived from a larger utility function with more urban economic variables. This will be discussed in Section 3.

Next, we will formulate the suppliers of apartments. Since the supply side plays a little role in this paper, we simplify this side of the model. Although we adopt the story of production, it can be translated into an exchange economy, which will be explained later.

The set of landlords is given as $N=\left\{1^{\prime}, \ldots, n^{\prime}\right\}$. We assume that each landlord $j \in N$ provides apartments in one category, say $k$, and has a cost function $C_{j}\left(y_{j}\right): \mathbf{Z}_{+} \rightarrow \mathbf{R}_{+}$with $C_{j}(0)=0$ and $C_{j}(1)>0$, where $\mathbf{Z}_{+}$is the set of nonnegative integers. The cost to provide $y_{j}$ units of apartments of the $k$ th category is $C_{j}\left(y_{j}\right)$. No fixed costs are required when no units are provided to the market.

As far as competitive equilibrium is concerned, we can assume without loss of generality that only one landlord $j$ provides apartments in the $k$ th category. Hence, the set $N$ becomes $\left\{1^{\prime}, \ldots, T^{\prime}\right\}$, and landlord $k \in N$ is the only landlord providing the $k$ th apartments. The reason for this reduction is as follows: Let $N_{k}$ be the set of landlords in the original $N$ to provide some units of the $k$ th category. We can combine the cost functions $\left\{C_{j}\right\}_{j \in N_{k}}$ into the one cost function $C_{k}: \mathbf{Z}_{+} \rightarrow \mathbf{R}_{+}$defined by

$$
C_{k}\left(y_{k}\right)=\min \left\{\sum_{j \in N_{k}} C_{j}\left(y_{j}\right): \sum_{j \in N_{k}} y_{j}=y_{k} \text { and } y_{j} \in \mathbf{Z}_{+} \text {for all } j \in N_{k}\right\}
$$

for each $y_{k} \in \mathbf{Z}_{+}$. This $k$ is regarded as the representative landlord providing the $k$ th apartments. ${ }^{2}$ From now on, $N$ is assumed to be $\left\{1^{\prime}, \ldots, T^{\prime}\right\}$, and landlord $k \in N$ is the only landlord providing the $k$ th apartments.

We are dealing with the problem of dwellings. Thus, the cost function represents operating costs. The marginal cost $C_{k}\left(y_{k}+1\right)-C_{k}\left(y_{k}\right)$ is the operating cost newly incurred for the landlord when this additional unit is leased to a household. If the rent is higher than $C_{k}\left(y_{k}+1\right)-C_{k}\left(y_{k}\right)$, then it would be better to lease this unit, but otherwise, it would be better to keep the unit.

We assume:

Assumption B (Convexity). For each $k \in N$,

$$
C_{k}\left(y_{k}+1\right)-C_{k}\left(y_{k}\right) \leqslant C_{k}\left(y_{k}+2\right)-C_{k}\left(y_{k}+1\right) \quad \text { for all } y_{k} \in \mathbf{Z}_{+} .
$$

This is a discrete version of the standard convexity assumption on a cost function, and means that the marginal cost of providing one additional unit is (weakly) increasing.

\footnotetext{
2 This means that as far as the price taker behavior is assumed, "many landlords" can be reduced into "one landlord." Of course, the justification of price-taker behavior itself needs the assumption of "many landlords." However the assumption of one landlord for one category simplifies our notation.
} 
Under these assumptions, we have a competitive equilibrium. Let $(p, x, y)=\left(\left(p_{1}, \ldots, p_{T}\right)\right.$, $\left.\left(x_{1}, \ldots, x_{m}\right),\left(y_{1}, \ldots, y_{T}\right)\right)$ be a triple of $p \in \mathbf{R}_{+}^{T}, x \in\left\{\mathbf{e}^{0}, \mathbf{e}^{1}, \ldots, \mathbf{e}^{T}\right\}^{m}$ and $y \in \mathbf{Z}_{+}^{T}$. We say that $(p, x, y)$ is a competitive equilibrium iff

Utility Maximization under the Budget Constraint. For all $i \in M$,

(1) $I_{i}-p x_{i} \geqslant 0$, where $p x_{i}=\sum_{k=1}^{T} p_{k} x_{i k}$;

(2) $u_{i}\left(x_{i}, I_{i}-p x_{i}\right) \geqslant u_{i}\left(x_{i}^{\prime}, I_{i}-p x_{i}^{\prime}\right)$ for all $x_{i}^{\prime} \in\left\{\mathbf{e}^{0}, \mathbf{e}^{1}, \ldots, \mathbf{e}^{T}\right\}$ with $I_{i}-p x_{i}^{\prime} \geqslant 0$.

Profit Maximization. For all $k=1, \ldots, T, p_{k} y_{k}-C_{k}\left(y_{k}\right) \geqslant p_{k} y_{k}^{\prime}-C_{k}\left(y_{k}^{\prime}\right)$ for all $y_{k}^{\prime} \in \mathbf{Z}_{+}$.

Balance of the Total Demand and Supply. $\sum_{i \in M} x_{i}=\sum_{k=1}^{T} y_{k} \mathbf{e}^{k}$.

These conditions constitute the standard notion of competitive equilibrium. Notice that the lefthand side of the Balancedness condition is the sum of $T$ dimensional vectors. However, since each $y_{k}$ of the right-hand side is an integer, we multiply it with the unit vector $\mathbf{e}^{k}$; thus both sides have the same dimension $T$.

The above housing market model is an extension of the "assignment market model" of Shapley-Shubik [21] in the two respects that we allow the utility functions $u_{i}$ to have income effects and that a landlord may provide more than one unit of apartments. The former respect is crucial in this paper. Kaneko [14] and Kaneko-Yamamoto [13] proved the existence of a competitive equilibrium for this housing market model. ${ }^{3}$

Theorem 2.1 (Existence). There is a competitive equilibrium $(p, x, y)$ in $(M, N)$.

The purpose of this paper is to study not general properties of equilibrium in $(M, N)$ but more specific behavior of a competitive rent vector. Specifically, we target to study comparative statics. For this reason, we need to specify our housing model more.

We say that $p=\left(p_{1}, \ldots, p_{T}\right)$ is a competitive rent vector iff $(p, x, y)$ is a competitive equilibrium for some $x \in\left\{\mathbf{e}^{0}, \mathbf{e}^{1}, \ldots, \mathbf{e}^{T}\right\}^{m}$ and $y \in \mathbf{Z}_{+}^{T}$. Also, we say that $p=\left(p_{1}, \ldots, p_{T}\right)$ is a maximal competitive rent vector iff $p \geqslant p^{\prime}$ for any competitive rent vector $p^{\prime}$. A competitive equilibrium $(p, x, y)$ is called a maximal competitive equilibrium iff $p$ is maximal. In the parallel manner, we can define a minimal competitive rent vector and a minimal competitive equilibrium. In this paper, we will focus on a maximal competitive rent vector, though we can adopt a minimal competitive rent vector for our study of comparative statics.

By definition, a maximal competitive rent vector would be unique if it ever exists. In a standard equilibrium model, the existence of such a price vector could not be expected. However, we have the existence of a maximal competitive rent vector in $(M, N)$. This fact has been known in slightly different models since the pioneering work of Shapley-Shubik [21] and GaleShapley [8]. A result close to the following theorem is found in Kaneko [15] and Miyake [17]. ${ }^{4}$ The proof of this theorem will be provided upon request.

\footnotetext{
3 A similar existence result was obtained by Gerber [9], using a bid-offer model close to the literature of urban economics.

4 In the literature of assignment games, it is shown that the core of a two-sided assignment market has the specific geometric structure that the core has the maximal and minimal payoff vectors for one side of players. Also, it holds that the core is equivalent to the set of competitive allocations in a typical assignment market, though this equivalence
} 
Theorem 2.2 (Existence of a Maximal Competitive Rent Vector). There is a maximal competitive price vector in the housing market model $(M, N)$.

It is also shown in the same manner that a minimal competitive rent vector exists. Nevertheless, we will use only the maximal one in the subsequent discussions.

\subsection{Specific assumptions on the rental housing market $(M, N)$}

In this section, we give more specific assumptions particularly on the utility functions of the households in order to facilitate our study of comparative statics.

First, we order the households by the order of incomes, that is,

$$
I_{1} \geqslant I_{2} \geqslant \cdots \geqslant I_{m}
$$

This is simply a renaming of households.

The following is a substantive restriction:

Assumption C (Identical Utility Function). $u_{i}(\cdot, \cdot)=u_{i^{\prime}}(\cdot, \cdot)$ for all $i, i^{\prime} \in M$.

We denote $u_{i}\left(x_{i}, c\right)$ by $u\left(x_{i}, c\right)$ by eliminating the subscript $i$. Households may still behave differently since they have different incomes and since the utility function $u\left(x_{i}, c\right)$ may admit income effects. In the context of urban economics, Assumption $\mathrm{C}$ is interpreted as meaning that every household commutes to the same office area. It excludes the situation with two or more office districts to one of which each household commutes. Indeed, when a housing market has two or more office districts, only the households who commute to the same business district have identical utility functions. This extension will be discussed in Section 8.

Assumption $\mathrm{C}$ is almost indispensable in that without it, our comparative statics results would be too complicated to be operational. We may extend some of our results to an economy without Assumption C, which will be remarked in Section 8.

Some general theorists may criticize Assumption $\mathrm{C}$ as too restrictive. However, the aim of this paper is not to discuss general properties such as the existence and/or Pareto optimality of an equilibrium, but to study more concrete properties. From the viewpoint of such a research, if there are no positive reasons for differences in utility functions, it would be better to assume the identical utility function among the households of commuting to the same business district.

We impose also the following two assumptions on $u(\cdot, \cdot)$.

Assumption D (Substitutability). If $u\left(x_{i}, c\right)>u\left(x_{i}^{\prime}, c^{\prime}\right)$, then $u\left(x_{i}, c\right)=u\left(x_{i}^{\prime}, c^{\prime}+\delta\right)$ for some $\delta>0$.

Assumption E (Normality). If $u\left(x_{i}, c\right)=u\left(x_{i}^{\prime}, c^{\prime}\right)$ and $c<c^{\prime}$, then $u\left(x_{i}, c+\delta\right)>u\left(x_{i}^{\prime}, c^{\prime}+\delta\right)$ for any $\delta>0$.

Assumption D may be interpreted as meaning that the variety of apartments in $(M, N)$ is not so large that the difference between any two apartments may be compensated by an increase

requires each landlord to produce at most one unit in the model of the present paper (see Kaneko [14]). The existence of a maximal rent vector is expected from these facts. Indeed, our housing market keeps this property for the competitive rent vectors. 
in consumption. Assumption $\mathrm{E}$ is the normality assumption on the qualities of apartments. Its premise states that the apartment $x_{i}$ is better than $x_{i}^{\prime}$, and the claim is that if consumption increases by the same magnitude $\delta$, a better apartment is preferred to a worse one. Assumption $\mathrm{E}$ will play a central role in the study of this paper.

Assumption $\mathrm{E}$ is the same as the standard normality assumption in that when the income of a household increases, his demand shifts to a better apartment or remains the same. This will be stated as the property of a competitive equilibrium in Lemma 2.3(2), rather than the demand function. Indeed, the assertion can be rewritten for the demand function.

The other direction of $\delta$ in Assumption E holds: If $u\left(x_{i}, c\right)=u\left(x_{i}^{\prime}, c^{\prime}\right)$ and $c<c^{\prime}$, then $u\left(x_{i}, c-\delta\right)<u\left(x_{i}^{\prime}, c^{\prime}-\delta\right)$ for any $\delta(c \geqslant \delta>0)$. Indeed, we can prove its contrapositive as follows: Let $c<c^{\prime}$ and $u\left(x_{i}, c-\delta\right) \geqslant u\left(x_{i}^{\prime}, c^{\prime}-\delta\right)$ for some $\delta(c \geqslant \delta>0)$. Then, by Assumption $\mathrm{D}$, we have $u\left(x_{i}, c-\delta\right)=u\left(x_{i}^{\prime}, c^{\prime}-\delta+\delta^{\prime}\right)$ for some $\delta^{\prime} \geqslant 0$. Since $c-\delta<c^{\prime}-\delta+\delta^{\prime}$, we have $u\left(x_{i}, c\right)>u\left(x_{i}^{\prime}, c^{\prime}+\delta^{\prime}\right) \geqslant u\left(x_{i}^{\prime}, c^{\prime}\right)$ by Assumptions $\mathrm{E}$ and $\mathrm{A}$.

We assume that the apartments are ordered as follows:

Assumption F (Qualities of Apartments). $u\left(\mathbf{e}^{1}, 0\right)>u\left(\mathbf{e}^{2}, 0\right)>\cdots>u\left(\mathbf{e}^{T}, 0\right)$.

Under Assumption F, the qualities of the apartments are unambiguously defined. Nevertheless, this is to avoid unnecessary complications in the presentations of our results and arguments for them. If we replace the strict inequalities by weak inequalities, this would be simply a renaming of the apartments. It is only a restriction that there are no indifferences in $\left(\mathbf{e}^{1}, 0\right), \ldots,\left(\mathbf{e}^{T}, 0\right)$.

Note that Assumption F together with Assumptions A, D and E imply:

$$
u\left(\mathbf{e}^{1}, c\right)>u\left(\mathbf{e}^{2}, c\right)>\cdots>u\left(\mathbf{e}^{T}, c\right) \text { for all } c \geqslant 0 .
$$

This is verified as follows: Since $u\left(\mathbf{e}^{t}, c\right) \geqslant u\left(\mathbf{e}^{t}, 0\right)>u\left(\mathbf{e}^{t+1}, 0\right)$ by Assumptions A and F, we have, by Assumption $\mathrm{D}, u\left(\mathbf{e}^{t}, 0\right)=u\left(\mathrm{e}^{t+1}, d\right)$ for some $d>0$. By Assumptions $\mathrm{E}$ and $\mathrm{A}$, we have $u\left(\mathbf{e}^{t}, c\right)>u\left(\mathbf{e}^{t+1}, c+d\right)>u\left(\mathbf{e}^{t+1}, c\right)$. Thus, (2.2) is equivalent to Assumption $\mathrm{F}$. This fact will be used without reference.

Note that Assumption F or (2.2) does not mean that the apartments are ordered by the distance to the central office district. In fact, the above model has a large freedom to include various urban structures. A numerical example for our rental housing market will be discussed in Section 3 and Section 7.

Some reader may think that the combination of the identical utility function (Assumption C) and normality assumption (Assumption E) could be more restrictive than what appear, in applications of our model to more concrete urban economies. For example, it might be suspected that the combination could imply separability of urban economic variables in the utility function. We will consider two examples about this problem in the end of Section 3.

\subsection{The rent equation and the differential rent vector}

We will provide a system of equations, which we will call the rent equation. The rent equation determines a unique rent vector if a boundary condition, e.g., a rent for the marginal category, is given. We will call this vector a differential rent vector and will directly consider a differential rent vector rather than the maximal competitive rent vector in the subsequent discussions. In this section, we will give certain sufficient conditions for the maximal competitive rent vector to be a differential rent vector. 
To define the rent equation, we need some lemmas, a definition and an additional assumption. First, we show that in equilibrium, (1) a better apartment is priced higher than a worse one; and (2) a richer household rents an apartment of a better or equal quality. As stated after Assumption $\mathrm{E}$, the second assertion is interpreted as meaning that the apartment qualities are regarded as normal goods, though it is not stated in terms of the demand function.

Lemma 2.3. Let $(p, x, y)$ be any competitive equilibrium.

(1) If $k^{\prime}<k$ and $x_{i}=\mathbf{e}^{k}$ for some $i$, then $p_{k^{\prime}}>p_{k}$;

(2) If $x_{i}=\mathbf{e}^{k}, x_{i^{\prime}}=\mathbf{e}^{k^{\prime}}$ and $I_{i}>I_{i^{\prime}}$, then $k \leqslant k^{\prime}$.

Proof. We prove only (2), and can prove (1) similarly. Let $I_{i}>I_{i^{\prime}}$. Suppose, on the contrary, $k>k^{\prime}$. Then $p_{k^{\prime}}>p_{k}$ by (1). Since $u\left(\mathbf{e}^{k^{\prime}}, I_{i^{\prime}}-p_{k^{\prime}}\right) \geqslant u\left(\mathbf{e}^{k}, I_{i^{\prime}}-p_{k}\right)$ by utility maximization, we have $u\left(\mathbf{e}^{k^{\prime}}, I_{i^{\prime}}-p_{k^{\prime}}\right)=u\left(\mathbf{e}^{k}, I_{i^{\prime}}-p_{k}+\delta^{\prime}\right)$ for some $\delta^{\prime} \geqslant 0$ by Assumption D. Let $\delta^{\prime \prime}=I_{i}-I_{i^{\prime}}$. Since $I_{i^{\prime}}-p_{k^{\prime}}<I_{i^{\prime}}-p_{k} \leqslant I_{i^{\prime}}-p_{k}+\delta^{\prime}$ by $p_{k^{\prime}}>p_{k}$, we have $u\left(\mathbf{e}^{k^{\prime}}, I_{i^{\prime}}-p_{k^{\prime}}+\delta^{\prime \prime}\right)>u\left(\mathbf{e}^{k}, I_{i^{\prime}}-\right.$ $\left.p_{k}+\delta^{\prime}+\delta^{\prime \prime}\right)$ by Assumption E. Hence, we have $u\left(\mathbf{e}^{k^{\prime}}, I_{i}-p_{k^{\prime}}\right)=u\left(\mathbf{e}^{k^{\prime}}, I_{i^{\prime}}-p_{k^{\prime}}+\delta^{\prime \prime}\right)>$ $u\left(\mathbf{e}^{k}, I_{i^{\prime}}-p_{k}+\delta^{\prime}+\delta^{\prime \prime}\right)=u\left(\mathbf{e}^{k}, I_{i}-p_{k}+\delta^{\prime}\right) \geqslant u\left(\mathbf{e}^{k}, I_{i}-p_{k}\right)$, where the two equalities follow from $\delta^{\prime \prime}=I_{i}-I_{i^{\prime}}$ and the last inequality follows from Assumption A. This is a contradiction to utility maximization for household $i$ at $x_{i}=\mathbf{e}^{k}$.

In the assertion (1) of Lemma 2.3, category $k^{\prime}$ may be inactive in the sense that no units are traded, while category $k$ is active. We rule out such a case, that is, we assume that there is a category $f$ dividing the apartments into the active categories and inactive ones: For any maximal competitive equilibrium $(p, x, y)$,

Assumption G. $y_{k}>0$ for $k=1, \ldots, f$ and $y_{k}=0$ for $k=f+1, \ldots, T$.

We call $f$ the marginal category. Then, by Lemma $2.3(1)$, we have the following inequalities:

$$
p_{1}>p_{2}>\cdots>p_{f} \text {. }
$$

By Lemma 2.3(2), we can assume without loss of generality that the households go to categories $1, \ldots, T$ in the order of their incomes. That is, the highest income group is in category 1 , the second highest income group is in category 2, and so on. Assumption E (normality) is crucial for this step.

We find the household with the lowest income in each active category. This household is called the boundary household of this category. We introduce a function to express the boundary households. Let $(p, x, y)$ be a maximal competitive equilibrium. We define the function $G(k)(k=1, \ldots, T)$ by

$$
G(k)=\sum_{t=1}^{k} y_{t} \quad \text { for } k=1, \ldots, T .
$$

This $G(k)$ is the accumulated number of units up to the $k$ th category. When $k \leqslant f, G(k)$ corresponds to the household with the lowest income in the group of category $k$, i.e., he is the boundary household in category $k$. When $k>f$, there are no corresponding boundary households in category $k$.

Although the maximal competitive rent vector is unique, there may be multiple maximal competitive allocations. The boundary household $G(k)$ in each category depends upon the choice of 
a maximal competitive allocation, but this does not cause any problem because of the uniqueness of the maximal competitive rent vector.

Now, we can present the main tool for the subsequent discussions.

Definition 2.4. We call the following system of equations the rent equation:

$$
\begin{aligned}
u\left(\mathbf{e}^{f-1}, I_{G(f-1)}-r_{f-1}\right) & =u\left(\mathbf{e}^{f}, I_{G(f-1)}-r_{f}\right), \\
u\left(\mathbf{e}^{f-2}, I_{G(f-2)}-r_{f-2}\right) & =u\left(\mathbf{e}^{f-1}, I_{G(f-2)}-r_{f-1}\right) \\
& \vdots \\
u\left(\mathbf{e}^{1}, I_{G(1)}-r_{1}\right) & =u\left(\mathbf{e}^{2}, I_{G(1)}-r_{2}\right) .
\end{aligned}
$$

These equations have unknown variables $r_{1}, \ldots, r_{f}$. We say that $\left(r_{1}, \ldots, r_{f}\right)$ is a differential rent vector iff it is a solution of the rent equation. Under our assumptions, if $r_{f}$ is given with $u\left(\mathbf{e}^{1}, 0\right)<u\left(\mathbf{e}^{f}, I_{G(f-1)}-r_{f}\right)$, then $\left(r_{1}, \ldots, r_{f-1}\right)$ is uniquely determined, i.e., the uppermost equation gives $r_{f-1}$, and the second uppermost one gives $r_{f-2}$, and so on. We write this result as Lemma 2.5. However, when $r_{f}$ is not fixed, there are many differential rent vectors.

Lemma 2.5. Let $r_{f}^{*}$ be given with $u\left(\mathbf{e}^{1}, 0\right)<u\left(\mathbf{e}^{f}, I_{G(f-1)}-r_{f}^{*}\right)$. Then the rent equation has a unique solution $\left(r_{1}, \ldots, r_{f}\right)$ with $r_{f}=r_{f}^{*}$.

As already stated, the rent equation (2.5) is reminiscent of Ricardo's [19] theory of differential rents of farm lands around a city. It is argued that the absolute rent is determined by the agricultural productivity of the marginal land, and then that the rent of a land closer to the city is higher, reflecting its advantage in transportation to the city. A similar structure is found in (2.5). Suppose that the rent $r_{f}$ in the marginal category $f$ is given. The uppermost equation of (2.5) makes the boundary household $G(f-1)$ of category $f-1$ indifferent between the apartments in $f-1$ and $f$ at the rents $r_{f-1}$ and $r_{f}$. The advantage of $f-1$ over $f$ is reflected in the difference between $r_{f-1}$ and $r_{f}$. Since the utility function has income effects, richer people succeed in obtaining better apartments. The other equations of (2.5) are considered from the view points of the boundary households $G(f-2), \ldots, G(1)$.

A similar argument is found in the literature of urban economics: The land rents form an envelope curve of the bid-offer curves of individual households, e.g., Alonso [1], Muth [18], Braid [6] and Gerber [9]. The differential rent vector defined by (2.5) can be regarded also an envelope curve of the rent vectors determined by the boundary households $G(1), \ldots, G(f-1)$.

We can guarantee the maximal competitive rent vector to be a differential rent vector under certain conditions. These conditions look slightly restrictive, but differential rent vectors can be still regarded as good approximation of the maximal competitive rents. In the subsequent analysis, we will adopt a differential rent vector as a representative of a competitive rent vector.

Theorem 2.6 (Condition for the Rent Equation). Let $(p, x, y)=\left(\left(p_{1}, \ldots, p_{T}\right), x, y\right)$ be a maximal competitive equilibrium. If at least one of the following (1) and (2) holds:

(1) $I_{G(k)}=I_{G(k)+1}$ for each $k=1, \ldots, f-1$;

(2) $p_{k}<C_{k}\left(y_{k}+1\right)-C_{k}\left(y_{k}\right)$ for each $k=1, \ldots, f-1$,

then $\left(p_{1}, \ldots, p_{f}\right)$ is a differential rent vector, that is, the differential rent vector $\left(r_{1}, \ldots, r_{f}\right)$ determined by $r_{f}=p_{f}$ coincides with $\left(p_{1}, \ldots, p_{f}\right)$. 
A merit of this theorem is as follows: We take $p_{f}$ as given, which is endogenously determined by a maximal competitive equilibrium. Then, under conditions (1) and/or (2), the relevant part of the maximal competitive rent vector satisfies the rent equation (2.5). By the argument before Lemma 2.5, we can calculate the maximal competitive rents $p_{1}, \ldots, p_{f-1}$. In the application to real data in the Tokyo area in Section 7 , the rent $p_{f}$ of the marginal category $f$ is also estimated from the real data.

Condition (1) states that the income $I_{G(k)}$ of the last household in the $k$ th category coincides with the income $I_{G(k)+1}$ of the first household in the $(k+1)$ th category. When the number of households is large and their incomes are distributed in a (relatively) continuous manner, (1) holds approximately. In this case, a differential rent vector is an approximation of the maximal competitive rent vector.

Since $(p, x, y)$ is a competitive equilibrium, it hods generally that

$$
C_{k}\left(y_{k}\right)-C_{k}\left(y_{k}-1\right) \leqslant p_{k} \leqslant C_{k}\left(y_{k}+1\right)-C_{k}\left(y_{k}\right) .
$$

In addition to this, (2) requires the right inequality to hold strictly. According to the interpretation of a rental housing as a dwelling, apartments are already built and new units are costly. Condition (2) is naturally applied to this case. We will use this case in our application to numerical examples in Section 3 and Section 7.

It is easy to prove case (1), but (2) needs a longer proof. A proof of (2) will be given in Appendix A. Here, we consider only (1). Recall that $G(k)$ is the last household living in the $k$ th category and $G(k)+1$ is the first household living in the $(k+1)$ th category. It holds by utility maximization for households $G(k)$ and $G(k)+1$ that

$$
\begin{aligned}
& u\left(\mathbf{e}^{k}, I_{G(k)}-p_{k}\right) \geqslant u\left(\mathbf{e}^{k+1}, I_{G(k)}-p_{k+1}\right) \quad \text { and } \\
& u\left(\mathbf{e}^{k+1}, I_{G(k)+1}-p_{k+1}\right) \geqslant u\left(\mathbf{e}^{k}, I_{G(k)+1}-p_{k}\right) .
\end{aligned}
$$

By condition (1), these hold with equalities, i.e., the rent equation holds.

When neither (1) nor (2) holds, the maximal competitive rent vector may fail to satisfy the rent equation (2.5). This is shown by Example 2.7. Moreover, even under (2), a competitive rent vector may not satisfy the rent equation, but it holds generally that each left-hand side of (2.5) is greater than or equal to the corresponding right-hand side. It is the point of (2.5) for each line to hold with equality. Under condition (1), any competitive rent vector satisfies the rent equation (2.5). When the market is close to perfect competition, (1) holds approximately. Hence, a differential rent vector may be a good representative of a competitive rent vector.

We have emphasized the similarity of the rent equation to the Ricardian theory of differential rents of farm lands around a city. In the Ricardian theory, the rent of the marginal land is determined by agricultural productivity of the marginal land. Similar to this, the marginal category of apartments are slightly different from the other categories. The determination of market rent $p_{f}$ is related closely to the rent of farm land. For the category $k<f$, apartments are dwellings, and the marginal cost $C_{k}\left(t_{k}+1\right)-C_{k}\left(t_{k}\right)$ is interpreted as operating costs, which is typically smaller than the market rent $p_{k}$. However, in the category $f$, new apartments are possibly supplied by transforming farm lands into residential lands. In this case, $C_{f}\left(t_{f}+1\right)-C_{f}\left(t_{f}\right)$ is calculated as the monthly value of the production (+operation) cost. This remark will be relevant the treatment of $r_{f}$ in Sections 5 and 6.

Example 2.7. Suppose that there are only two households 1 and 2. Let their utility function $u(x, c)$ be given as

$$
u(x, c)=h_{k}+\sqrt{c} \quad \text { for } k=1,2,3,
$$


where $h_{1}=10, h_{2}=7$ and $h_{3}=3$. Also, let $I_{1}=100$ and $I_{2}=52$. Assume $p_{1}=51$ and $p_{2}=25$. Under these rents, household 1 rents apartment 1, and household 2 rents apartment 2 . Indeed,

$$
\begin{aligned}
u\left(\mathbf{e}^{1}, I_{1}-p_{1}\right) & =10+\sqrt{100-51}=17>u\left(\mathbf{e}^{2}, I_{1}-p_{2}\right) \\
& =7+\sqrt{100-25}=15.66 \ldots, \\
u\left(\mathbf{e}^{2}, I_{2}-p_{1}\right) & =10+\sqrt{52-51}=11<u\left(\mathbf{e}^{2}, I_{2}-p_{2}\right) \\
& =7+\sqrt{52-25}=14.19 \ldots
\end{aligned}
$$

Suppose that there are two landlords $1^{\prime}$ and $2^{\prime}$ such that $1^{\prime}$ has two units with valuation 51 for each unit and $2^{\prime}$ has two units with valuation 25 for each. Then the above competitive equilibrium is maximal and, in fact, is minimal, too. In this economy, the rent equation corresponds only to (2.6), which fails to hold with equality.

We are focussing on the maximal competitive rent vector and differential rent vectors for (2.5). Instead of the maximal one, we may focus on the minimal competitive rent vector. With the modification of (2.5) with the replacement of the boundary income $I_{G(k)}$ by $I_{G(k)+1}$ for $k=$ $1, \ldots, f-1$, the above argument could be carried over to the case of minimal competitive rents and differential rents. It follows from this consideration that under condition (1) of Theorem 2.6 holds, if the competitive rent $p_{f}$ for the marginal apartment is uniquely determined, then all the rents are uniquely determined. Incidentally, $p_{f}$ might be determined by the farm land rent, and it could be considered to be determined uniquely.

\section{Application to a rental housing market in Tokyo (1)}

In this section, we exemplify our theory by an application to a rental housing market in Tokyo. This application will be continued in Section 7.

Consider the JR (Japan Railway) Chuo line running from Tokyo station to the west direction, along which residential areas are spread out. See Fig. 3.1. The line has 24 stations from Tokyo station up to Hachioji station, which is almost on the west boundary of the Tokyo great metropolitan area. Here, we consider only a submarket of the entire market. That is, we take 5 stations and 4 types of sizes of apartments. Now, we explain how we formulate this submarket by means of a housing market model $(M, N)$.

Look at Table 3.1: We take 5 railway stations, near which four sizes of apartments are supplied. The first column of Table 3.1 shows the time distance from Tokyo station to each railway station, i.e., 18, 28, 35, 41 and 53 (minutes). It is assumed that people commute to Tokyo station (office area) from their apartments. The first row of Table 3.1 designates the sizes of apartments. These intervals are represented by the medians $15,35,55$ and $75\left(\mathrm{~m}^{2}\right)$, which we plug into a

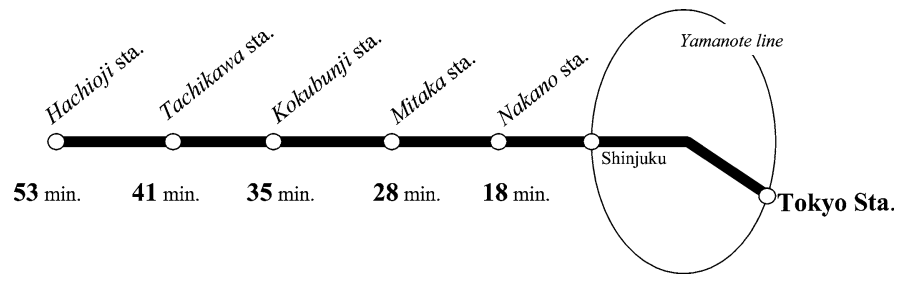

Fig. 3.1. Chuo line. 
Table 3.1

\begin{tabular}{|l|l|l|}
\hline$k$ & $h_{k}$ & $w_{k}$ \\
\hline
\end{tabular}

\begin{tabular}{|c|c|c|c|c|c|c|c|c|c|c|c|c|}
\hline \multirow{3}{*}{$\begin{array}{l}\text { Time (min) } \\
\text { 18:Nakano }\end{array}$} & \multicolumn{12}{|c|}{ Size $\left(m^{2}\right)$} \\
\hline & \multicolumn{3}{|c|}{$<25$} & \multicolumn{3}{|c|}{$25-45$} & \multicolumn{3}{|c|}{$45-65$} & \multicolumn{3}{|c|}{$65-85$} \\
\hline & 15 & 6.9 & 1204 & 10 & 68.9 & 780 & 5 & 130.9 & 250 & 1 & 192.9 & 113 \\
\hline 28:Mitaka & 17 & -15.1 & 608 & 12 & 46.9 & 391 & 7 & 108.9 & 197 & 2 & 170.9 & 63 \\
\hline 35:Kokubunji & 18 & -30.5 & 373 & 13 & 31.5 & 229 & 8 & 93.5 & 77 & 3 & 155.5 & 20 \\
\hline 41:Tachikawa & 19 & -43.7 & 362 & 14 & 18.3 & 316 & 9 & 80.3 & 91 & 4 & 142.3 & 30 \\
\hline 53:Hachioji & 20 & -70.1 & 689 & 16 & -8.1 & 234 & 11 & 53.9 & 137 & 6 & 115.9 & 30 \\
\hline
\end{tabular}

utility function. ${ }^{5}$ Thus, the apartments are classified into $T=5 \times 4=20$ categories. When we consider data of apartment supplies, each apartment is classified into one category.

Before having the utility function $u: X=\left\{\mathbf{e}^{0}, \mathbf{e}^{1}, \ldots, \mathbf{e}^{20}\right\} \times R_{+} \rightarrow R$ in the sense of Section 2.2, we assume that the households have the common basic utility function as follows:

$$
\widehat{U}(t, s, c)=-2.2 t+3.1 s+80 \sqrt{c},
$$

where $t$ takes possible values $18,28,35,41,53$ and $s$ takes values $015,35,55,75$, and $c$ is the expenditure to consumption measured by thousand yen, e.g., if $c$ is 100,000 yen, then $c=100$. Calculating $-2.2 t+3.1 s$, we have $h_{k}(k=1, \ldots, 20)$ in the legend \begin{tabular}{|l|l|l|l|}
\hline$k$ & $h_{k}$ & $w_{k}$ \\
of Table 3.1. The
\end{tabular} first $k$ representing the quality rank of a category is determined by $-2.2 t+3.1 s$. For example, the term $-2.2 t+3.1 s$ takes the largest value at $(t, s)=(18,75)$, and thus, the label of the category is $k=1$. Up to $k=4$, this order follows the distance to Tokyo Station. But it takes the 5th value at $(t, s)=(18,55)$ and the 6 -value at $(53,75)$, where it is more important to decrease the commuting time than to have a larger size of an apartment.

The labeling of categories is denoted by the first number $k$ of the each cell of Table 3.1. Based on this correspondence, we define $u: X \rightarrow R$

$$
u\left(\mathbf{e}^{k}, c\right)=h_{k}+80 \sqrt{c},
$$

where $h_{k}=-2.2 t+3.1 s$ for $k \geqslant 1$ and $h_{0}$ is assumed to be -500 , i.e., $u\left(\mathbf{e}^{0}, c\right)=h_{0}+80 \sqrt{c}=$ $-500+80 \sqrt{c}$.

In the formulation given in Section 2.1, household $i$ chooses $k$ to maximize his utility $u\left(\mathbf{e}^{k}, I_{i}-p_{k}\right)$ with $I_{i} \geqslant p_{k}$. From the viewpoint of the basic utility function of (3.1), this can be expressed as the choice of $(t, s)$ to maximize

$$
\widehat{U}\left(t, s, I_{i}-p_{k(t, s)}\right)=-2.2 t+3.1 s+80 \sqrt{I_{i}-p_{k(t, s)}} \quad \text { with } I_{i} \geqslant p_{k(t, s)},
$$

where $k(t, s)$ is the category corresponding to $(t, s) .^{6,7}$

The derived utility function in (3.2) satisfies Assumptions A and C-F. The concavity of $80 \sqrt{c}$ corresponds to the normality assumption (Assumption E).

The third entry $w_{k}$ of the legend of Table 3.1 is the number of units for sale listed in the housing information service, the Yahoo Real Estate (15, Dec. 2004). Suppose that only those

\footnotetext{
5 The first interval is taken from 5 to 25 . In the Japanese standard, one-room apartments are categorized into this class.

6 If $-2.2 t+3.1 s=-2.2 t^{\prime}+3.1 s^{\prime}$, then it suffices to some tie-breaking rule.

7 In Japan, commuting costs are usually provided by a company. Therefore, commuting costs are not included in the budget constraint of a household. However, this custom is not necessarily found in other countries. The other case was analyzed more typically in the urban economics literature, such as Wheaton [27], Hartwick et al. [10], Braid [5] and Arnott et al. [3]. Haurin [11] and White [28] discussed the comparisons of surpluses for companies in different subsidy systems.
} 
units appear on the market and no units are newly built. Then, the cost function $C_{k}\left(y_{k}\right)$ may be formulated as

$$
C_{k}\left(y_{k}\right)= \begin{cases}c_{k} y_{k} & \text { if } y_{k} \leqslant w_{k} \\ \text { "large" } & \text { if } y_{k}>w_{k},\end{cases}
$$

where "large" is a number greater than $I_{1}$. That is, landlord $k$ has the reservation price (operating $\operatorname{cost}) c_{k}$ for all units he provides, but the cost to build a new unit is very large relative to this housing market. This satisfies Assumption B.

The number $w_{k}$ of apartment units in category $k(k=1, \ldots, 20)$ for sale is taken for a given period of time, say, two weeks, from the Yahoo Real Estate. These units will disappear from the market after that period. We assume that the same number of households are coming to the market to look for apartments, and that all the units are traded in the end of the period. Therefore, the total number $m$ of households on the market is $\sum_{k=1}^{20} w_{k}=6194$.

The remaining element of the housing market model $(M, N)$ is the incomes for the households. We assume that the (monthly) income distribution over $M=\{1, \ldots, m\}=\{1, \ldots, 6194\}$ is a uniform distribution from 100,000 yen to 900,000 yen. Hence, $I_{6194}=100,000$ and $I_{1}=900,000$. In fact, this uniform distribution is just for calculation, and can be changed into other distributions. ${ }^{8}$

Under the above specification of the housing market model $(M, N)$, we can calculate the differential rent vector $r=\left(r_{1}, \ldots, r_{20}\right)$ as in Table 3.2. This rent vector $r=\left(r_{1}, \ldots, r_{20}\right)$ is determined by the rent equation (2.5) with $r_{20}=49.6$. In Section 7.1, we will explain how to choose this $r_{20}=49.6$ and the particular utility function given by (3.1).

Table 3.2 gives the average rents $\bar{p}=\left(\bar{p}_{1}, \ldots, \bar{p}_{20}\right)$ of the data from the Yahoo Real Estate. In Fig. 3.2, $r=\left(r_{1}, \ldots, r_{20}\right)$ and $\bar{p}=\left(\bar{p}_{1}, \ldots, \bar{p}_{20}\right)$ are depicted. The evaluation of the discrepancy between $r$ and $\bar{p}$ will be discussed in Section 7.1.

In the above numerical example, we have assumed the separable utility function of (3.2) (or (3.1)), but our theory itself does not restrict to such a separable utility function. For example, we can modify our utility function as follows:

$$
u\left(e^{k}, c\right)=h_{k}+a(L-k) \sqrt{c},
$$

where $a$ and $L$ are positive constants. This does satisfy Assumption E. In this utility function, an apartment in a better category enhances the utility from consumption. However, Assumption E is violated if it is modified to:

$$
u\left(e^{k}, c\right)=h_{k}+a(L-t(k)) \sqrt{c},
$$

\begin{tabular}{|c|c|c|c|c|c|c|c|c|c|c|c|c|}
\hline \multirow{3}{*}{$\begin{array}{l}\text { Time (min) } \\
18 \text { : Nakano }\end{array}$} & \multicolumn{12}{|c|}{ Size $\left(m^{2}\right)$} \\
\hline & \multicolumn{3}{|c|}{$<25$} & \multicolumn{3}{|c|}{$25-45$} & \multicolumn{3}{|c|}{$45-65$} & \multicolumn{3}{|c|}{$65-85$} \\
\hline & 15 & 77.1 & 73.9 & 10 & 112.6 & 112.1 & 5 & 153.0 & 162.4 & 1 & 194.2 & 209.5 \\
\hline 28 : Mitaka & 17 & 67.4 & 69.3 & 12 & 99.4 & 101.6 & 7 & 138.6 & 141.3 & 2 & 179.7 & 183.9 \\
\hline 35 : Kokubunji & 18 & 61.7 & 58.8 & 13 & 90.6 & 82.6 & 8 & 128.6 & 118.3 & 3 & 169.5 & 149.3 \\
\hline 41 : Tachikawa & 19 & 57.3 & 60.1 & 14 & 83.2 & 78.1 & 9 & 120.0 & 118.8 & 4 & 160.7 & 129.6 \\
\hline 53 : Hachioji & 20 & 49.6 & 54.6 & 16 & 70.4 & 73.7 & 11 & 103.6 & 105.1 & 6 & 143.2 & 122.1 \\
\hline
\end{tabular}

Table 3.2

\begin{tabular}{|l|l|l|}
\hline$k$ & $r_{k}$ & $\bar{p}_{k}$ \\
\hline
\end{tabular}

\footnotetext{
8 Even if we change it to a truncated normal distribution, the calculated rents are not changed a lot.
} 


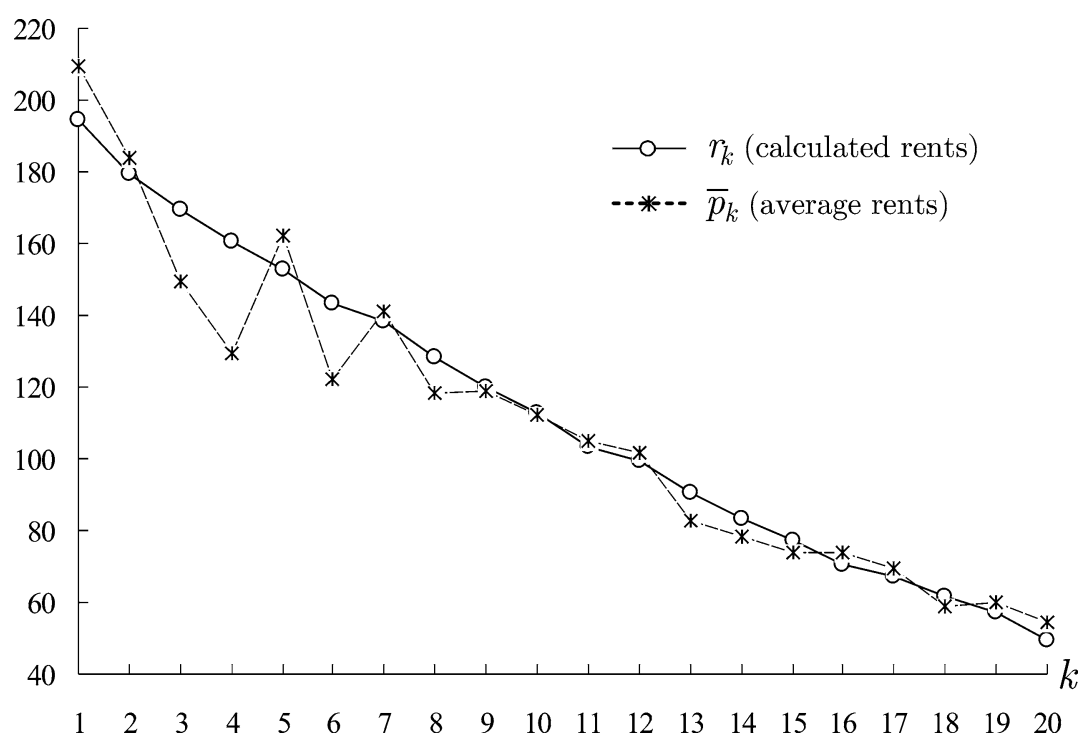

Fig. 3.2. Comparison.

where $t(k)$ is the commuting time (minutes) to $k$. In this example, an apartment in a closer location to the city center enhances the utility from consumption. One may think that "if a pleasant downtown life calls for high spending, low income households may concentrate more on the sizes and high income households more on the location of the apartment when judging its quality."9 This phenomenon is excluded from our analysis with Assumption E.

\section{The basic comparative statics theorem}

This section will formulate our comparative statics and will provide one basic theorem. This theorem will be crucial in obtaining comparative statics results in Sections 5 and 6. We remark that a particular comparative statics is not our focus; instead, we will focus on certain general properties of the behavior of rent vectors when some changes occur in parameters. ${ }^{10}$ Keeping this remark in mind, first we explain our comparative statics.

We will consider the behavior of a differential rent vector $r=\left(r_{1}, \ldots, r_{f}\right)$ defined by (2.5) when some parameters of the housing market $(M, N)$ change. Recall that a differential rent vector $r=\left(r_{1}, \ldots, r_{f}\right)$ is the representative of the maximal rent vector $p=\left(p_{1}, \ldots, p_{T}\right)$ as discussed in Section 2.3. We denote the market after those parameter changes by $(\widehat{M}, \widehat{N})$. Throughout the following, we make Assumptions A-G on both $(M, N)$ and $(\widehat{M}, \widehat{N})$. The unchanged part and the parameters changes are as follows:

(0) the apartment categories $1, \ldots, T$ and the utility function $u(\cdot, \cdot)$ remain the same;

(1) the households $M=\{1, \ldots, m\}$ change into $\widehat{M}=\{1, \ldots, \widehat{m}\}$, and the incomes $I_{1}, \ldots, I_{m}$ change into $\hat{I}_{1}, \ldots, \hat{I}_{\hat{m}}$;

\footnotetext{
9 This is pointed out by a referee.

10 Representative papers in the standard comparative statics studies are Wheaton [27], Hartwick et al. [10] and ArnottMacKinnon-Wheaton [4].
} 
(2) the landlords $N=\left\{1^{\prime}, \ldots, T^{\prime}\right\}$ remains the same, but the cost functions $C_{k}$ change into $\widehat{C}_{k}(k=1, \ldots, T)$.

A typical comparative statics takes a form of cause-effect in changes in one parameter and the rents. For example in the urban economics literature, various forms of comparative statics are found in, for example, Kaneko [15], Fujita [7, Section 3.6], and Braid [5,6]. We will not consider this form of a particular cause-effect comparative statics, but will consider general properties of the behavior of a differential rent vector when the housing market $(M, N)$ changes into $(\widehat{M}, \widehat{N})$. For this consideration, the relation between the rent $r_{k}$ and the boundary income $I_{G(k)}$ of category $k$ plays crucial roles. Incidentally, the changes in the boundary incomes $I_{G(1)}, \ldots, I_{G(f-1)}$ may be caused by ones in (1)-(2). We write the relevant changes explicitly:

(3) the marginal category $f$ changes into $\hat{f}$;

(4) the boundary income $I_{G(k)}$ changes into $\hat{I}_{\widehat{G}(k)}$.

The boundary household $\widehat{G}(k)$ may differ from $G(k)$.

In general, the income difference $\hat{I}_{\widehat{G}(k)}-I_{G(k)}$ is intimately related to the rent difference $\hat{r}_{k}-r_{k}$, and these differences play the major role in our study. The following theorem is basic, and also is suggestive for why we focus on income differences $\hat{I}_{\widehat{G}(k)}-I_{G(k)}$ rather than other parameters. This is a weaker form of the theorem given in a more specific environment in Kaneko [15, p. 39, Theorem 5(i)]. ${ }^{11}$

Theorem 4.1 (Basic Comparative Statics Theorem). Let $r=\left(r_{1}, \ldots, r_{f}\right)$ and $\hat{r}=\left(\hat{r}_{1}, \ldots, \hat{r}_{\hat{f}}\right)$ be differential rent vectors in $(M, N)$ and $(\widehat{M}, \widehat{N})$. Let $k$ be a category with $1 \leqslant k \leqslant \min (f, \hat{f})-1$. Then

$$
\hat{I}_{\widehat{G}(k)}-I_{G(k)} \leqslant \hat{r}_{k}-r_{k} \text { if and only if } \hat{r}_{k}-r_{k} \leqslant \hat{r}_{k+1}-r_{k+1} .
$$

Note that $\leqslant$ in (4.1) can be replaced by $\geqslant,>,<$ or $=$.

The point of Theorem 4.1 is that the comparison of the rent differences at two neighboring categories $k$ and $k+1$ is reduced into that of the rent difference and the boundary income difference of category $k$. The following equivalent form may be easier to understand:

$$
\hat{I}_{\widehat{G}(k)}-\hat{r}_{k} \leqslant I_{G(k)}-r_{k} \text { if and only if } \hat{r}_{k}-\hat{r}_{k+1} \leqslant r_{k}-r_{k+1} \text {. }
$$

That is, consumption is smaller in the new situation than in the original if and only if the rent difference for those neighboring categories becomes smaller. Since category $k+1$ is the reference point to the boundary household $G(k)$, he compares his utility from an apartment in category $k$ with one in category $k+1$. The left-hand inequality of (4.2) means that his consumption in category $k$ decreases, a fortiori, his utility decreases. This is equivalent to the relative decrease of the rent difference, which is expressed by the right-hand side of (4.2). For this equivalence, the normality assumption (Assumption E) is essential.

From the above theorem, we will obtain duality results in comparative statics, which will be the subjects of Sections 5 and 6.

11 Braid [5] obtained a similar result (differential equation (6) on p. 293) in a short-run housing market with a continuum of housing qualities. Based on it, he considered various comparative statics results. 
Notice asymmetry between $k$ and $k+1$ in (4.1) in that only the right-hand side of (4.1) includes $k$ and $k+1$. This asymmetry is caused by the definition of the rent equation (2.5) with the maximal competitive rent vector. If we adopt the minimal competitive rent vector, we should have a comparison between $k-1$ and $k$ in the right-hand side of (2.5).

Proof of Theorem 4.1. (Only-If): Suppose $\hat{I}_{\widehat{G}(k)}-I_{G(k)} \leqslant \hat{r}_{k}-r_{k}$, i.e., $\hat{I}_{\widehat{G}(k)}-\hat{r}_{k} \leqslant I_{G(k)}-r_{k}$. Let $\delta=\left(I_{G(k)}-r_{k}\right)-\left(\hat{I}_{\widehat{G}(k)}-\hat{r}_{k}\right)$. Since $u\left(\mathbf{e}^{k}, \hat{I}_{\widehat{G}(k)}-\hat{r}_{k}\right)=u\left(\mathbf{e}^{k+1}, \hat{I}_{\widehat{G}(k)}-\hat{r}_{k+1}\right)$ by the rent equation (2.5) for $(\widehat{M}, \widehat{N})$, we have, by Assumption E,

$$
u\left(\mathbf{e}^{k}, \hat{I}_{\widehat{G}(k)}-\hat{r}_{k}+\delta\right) \geqslant u\left(\mathbf{e}^{k+1}, \hat{I}_{\widehat{G}(k)}-\hat{r}_{k+1}+\delta\right) .
$$

The left-hand side is written as $u\left(\mathbf{e}^{k}, I_{G(k)}-r_{k}\right)$, which equals to $u\left(\mathbf{e}^{k+1}, I_{G(k)}-r_{k+1}\right)$ by (2.5) for $(M, N)$. Hence,

$$
u\left(\mathbf{e}^{k+1}, I_{G(k)}-r_{k+1}\right) \geqslant u\left(\mathbf{e}^{k+1}, \hat{I}_{G(k)}-\hat{r}_{k+1}+\delta\right) .
$$

This and the monotonicity (Assumption A) of $u$ on consumption imply $I_{G(k)}-r_{k+1} \geqslant \hat{I}_{G(k)}-$ $\hat{r}_{k+1}+\delta$. That is, $\hat{r}_{k}-r_{k} \leqslant \hat{r}_{k+1}-r_{k+1}$.

(If): Suppose $\hat{I}_{\widehat{G}(k)}-I_{G(k)}>\hat{r}_{k}-r_{k}$. Let $\delta=\left(\hat{I}_{\widehat{G}(k)}-I_{G(k)}\right)-\left(\hat{r}_{k}-r_{k}\right)$. By the rent equation for $(M N)$, we have $u\left(\mathbf{e}^{k}, I_{i}-r_{k}\right)=u\left(\mathbf{e}^{k+1}, I_{i}-r_{k+1}\right)$, and then, by Assumption $\mathrm{E}$ and $\delta>0$, $u\left(\mathbf{e}^{k}, \bar{\equiv}{ }_{k}+\delta\right)>u\left(\mathbf{e}^{k+1}, I_{i}-r_{k+1}+\delta\right)$. Thus, we have

$$
\begin{aligned}
u\left(\mathbf{e}^{k+1}, I_{G(k)}-r_{k+1}+\delta\right) & <u\left(\mathbf{e}^{k}, I_{G(k)}-r_{k}+\delta\right) \\
& =u\left(\mathbf{e}^{k}, \hat{I}_{\widehat{G}(k)}-\hat{r}_{k}\right)=u\left(\mathbf{e}^{k+1}, \hat{I}_{\widehat{G}(k)}-\hat{r}_{k+1}\right),
\end{aligned}
$$

where the last equality follows from the rent equation for $(\widehat{M}, \widehat{N})$. By Assumption A, we have $I_{G(k)}-r_{k+1}+\delta<\hat{I}_{G(k)}-\hat{r}_{k+1}$, which is equivalent to $\hat{r}_{k}-r_{k}>\hat{r}_{k+1}-r_{k+1}$.

\section{Duality in the income and rent differences}

This section will provide two theorems on the relationship between the differences of boundary incomes and the differences of rents. These theorems will be considered in the two cases of the changes in the boundary incomes. In these two cases, the relationship in the boundary income differences and rent differences exhibits certain dual structures.

\subsection{Differences of the boundary incomes}

Throughout this section, we assume that differential rent vectors $r=\left(r_{1}, \ldots, r_{f}\right)$ and $\hat{r}=$ $\left(\hat{r}_{1}, \ldots, \hat{r}_{\hat{f}}\right)$ are chosen and fixed in $(M, N)$ and $(\widehat{M}, \widehat{N})$. Thus, the marginal categories $f, \hat{f}$ and boundary households $G(k)(k \leqslant f), \widehat{G}(k)(k \leqslant \hat{f})$ are determined. We also assume $f \leqslant \hat{f}$.

In the following, we will consider the two cases:

$$
\begin{aligned}
& \hat{I}_{\widehat{G}(1)}-I_{G(1)} \geqslant \hat{I}_{\widehat{G}(2)}-I_{G(2)} \geqslant \cdots \geqslant \hat{I}_{\widehat{G}(f-1)}-I_{G(f-1)}, \\
& \hat{I}_{\widehat{G}(1)}-I_{G(1)} \leqslant \hat{I}_{\widehat{G}(2)}-I_{G(2)} \leqslant \cdots \leqslant \hat{I}_{\widehat{G}(f-1)}-I_{G(f-1)} .
\end{aligned}
$$

The inequalities of (5.1) mean that the difference of the boundary incomes for a better category is larger than that for a worse category. Those of (5.2) are opposite inequalities. When the boundary incomes increase at a constant rate, (5.1) holds, and when they decrease at a constant rate, (5.2) 
holds. One may think that when incomes increase, (5.1) is more likely than (5.2). However, even when incomes increases, changes in the supplies and/or demands of apartments may make (5.2) hold-a numerical example will be given in Section 7.

Neither of (5.1) and (5.2) might be expected since the changes in the boundary income differences are caused by those in the basic parameters of $(M, N)$. The subsequent consideration could be applied to such a case by focussing on a segment of the sequence of the boundary income differences. Indeed, we divide the sequence

$$
\hat{I}_{\widehat{G}(1)}-I_{G(1)}, \hat{I}_{\widehat{G}(2)}-I_{G(2)}, \ldots, \hat{I}_{\widehat{G}(f-1)}-I_{G(f-1)}
$$

into the $k$ segments of the form:

$$
\begin{array}{r}
\left\{\hat{I}_{\widehat{G}(1)}-I_{G(1)}, \ldots, \hat{I}_{\widehat{G}\left(l_{1}\right)}-I_{G\left(l_{1}\right)}\right\}, \\
\left\{\hat{I}_{\widehat{G}\left(l_{1}+1\right)}-I_{G\left(l_{1}+1\right)}, \ldots, \hat{I}_{\widehat{G}\left(l_{2}\right)}-I_{G\left(l_{2}\right)}\right\}, \\
\vdots \\
\left\{\hat{I}_{\widehat{G}\left(l_{k-1}+1\right)}-I_{G\left(l_{k-1}+1\right)}, \ldots, \hat{I}_{\widehat{G}\left(l_{k}\right)}-I_{G\left(l_{k}\right)}\right\}
\end{array}
$$

so that each segment is alternatively ascending or weakly descending, i.e., for $t=1, \ldots, k-1$,

$$
\begin{aligned}
& \text { if } \hat{I}_{\widehat{G}\left(l_{t-1}+1\right)}-I_{G\left(l_{t-1}+1\right)}<\cdots<\hat{I}_{\widehat{G}\left(l_{t}\right)}-I_{G\left(l_{t}\right)} \text {, } \\
& \text { then } \hat{I}_{\widehat{G}\left(l_{t}+1\right)}-I_{G\left(l_{t}+1\right)} \geqslant \cdots \geqslant \hat{I}_{\widehat{G}\left(l_{t+1}\right)}-I_{G\left(l_{t+1}\right)} \text {; } \\
& \text { if } \hat{I}_{\widehat{G}\left(l_{t-1}+1\right)}-I_{G\left(l_{t-1}+1\right)} \geqslant \cdots \geqslant \hat{I}_{\widehat{G}\left(l_{t}\right)}-I_{G\left(l_{t}\right)} \text {, } \\
& \text { then } \hat{I}_{\widehat{G}\left(l_{t}+1\right)}-I_{G\left(l_{t}+1\right)}<\cdots<\hat{I}_{\widehat{G}\left(l_{t+1}\right)}-I_{G\left(l_{t+1}\right)} .
\end{aligned}
$$

This partition is obtained as follows: When $\hat{I}_{\widehat{G}(1)}-I_{G(1)}<\hat{I}_{\widehat{G}(2)}-I_{G(2)}$, we let $l_{1}$ as the largest number so that $\hat{I}_{\widehat{G}(1)}-I_{G(1)}<\cdots<\hat{I}_{\widehat{G}\left(l_{1}\right)}-I_{G\left(l_{1}\right)}$. Then, we let $l_{2}$ be the largest number so that $\hat{I}_{\widehat{G}\left(l_{1}+1\right)}-I_{G\left(l_{1}+1\right)} \geqslant \cdots \geqslant \hat{I}_{\widehat{G}\left(l_{2}\right)}-I_{G\left(l_{2}\right)}$, and so on. When $\hat{I}_{\widehat{G}(1)}-I_{G(1)} \geqslant \hat{I}_{\widehat{G}(2)}-I_{G(2)}$, we start with the weakly descending segment, and so on.

The subsequent results hold for each segment by regarding it as the sequence of (5.1) or (5.2). An example with two segments will be given in Section 7.

\subsection{Shapes of rent differences}

Now, we have the following theorem.

Theorem 5.1 (Upward and Downward Inheritance Theorem). Let $r$ and $\hat{r}$ be differential rent vectors in $(M, N)$ and $(\widehat{M}, \widehat{N})$. Let $k$ be an integer with $1 \leqslant k \leqslant f-1$.

(1) (Upward Inheritance): Under (5.1),

(a) $\hat{r}_{k+1}-r_{k+1}<\hat{r}_{k}-r_{k}$ implies $\hat{r}_{k}-r_{k}<\hat{r}_{k-1}-r_{k-1}<\cdots<\hat{r}_{1}-r_{1}$;

(b) $\hat{r}_{k}-r_{k}<\hat{r}_{k+1}-r_{k+1}$ implies $\hat{r}_{k+1}-r_{k+1}<\cdots<\hat{r}_{f}-r_{f}$.

(2) (Downward Inheritance): Under (5.2),

(a) $\hat{r}_{k}-r_{k}>\hat{r}_{k+1}-r_{k+1}$ implies $\hat{r}_{k+1}-r_{k+1}>\cdots>\hat{r}_{f}-r_{f}$;

(b) $\hat{r}_{k+1}-r_{k+1}>\hat{r}_{k}-r_{k}$ implies $\hat{r}_{k}-r_{k}>\hat{r}_{k-1}-r_{k-1}>\cdots>\hat{r}_{1}-r_{1}$. 
Under (5.1), if the rent difference in category $k$ is larger than that in category $k+1$, the same inequality is inherited in the inner direction, and if the opposite inequality holds, the inheritance goes in the outer direction. The results of (2) are dual to those of (1), as condition (5.2) is the dual of (5.1).

Proof of Theorem 5.1. We show (a) of (2). In the dual manner, we can prove the others. Let $l$ be an arbitrary category with $k \leqslant l \leqslant f-1$. It suffices to show that $\hat{r}_{l}-r_{l}>\hat{r}_{l+1}-r_{l+1}$ implies $\hat{r}_{l+1}-r_{l+1}>\hat{r}_{l+2}-r_{l+2}$. Suppose $\hat{r}_{l}-r_{l}>\hat{r}_{l+1}-r_{l+1}$. By Theorem 4.1, we have $\hat{I}_{\widehat{G}(l)}-I_{G(l)}>\hat{r}_{l}-r_{l}$. By (5.2) and $\hat{r}_{l}-r_{l}>\hat{r}_{l+1}-r_{l+1}$, we have

$$
\hat{I}_{\widehat{G}(l+1)}-I_{G(l+1)} \geqslant \hat{I}_{\widehat{G}(l)}-I_{G(l)}>\hat{r}_{l}-r_{l}>\hat{r}_{l+1}-r_{l+1} .
$$

It follows from this and Theorem 4.1 that $\hat{r}_{l+1}-r_{l+1}>\hat{r}_{l+2}-r_{l+2}$.

We have the following theorem on the rent shape as a consequence of Theorem 5.1, which exhibits also duality.

Theorem 5.2 (Rent Shape Theorem). Let $r$ and $\hat{r}$ be differential rent vectors in $(M, N)$ and $(\widehat{M}, \widehat{N})$.

(1) (Convex Shape): Under (5.1), there are $k_{1}$ and $k_{2}\left(1 \leqslant k_{1} \leqslant k_{2} \leqslant f\right)$ such that

(a) $\hat{r}_{1}-r_{1}>\cdots>\hat{r}_{k_{1}}-r_{k_{1}}$;

(b) $\hat{r}_{k_{1}}-r_{k_{1}}=\cdots=\hat{r}_{k_{2}}-r_{k_{2}}$;

(c) $\hat{r}_{k_{2}}-r_{k_{2}}<\cdots<\hat{r}_{f}-r_{f}$.

(2) (Concave Shape): Under (5.2), there are $k_{1}$ and $k_{2}\left(1 \leqslant k_{1} \leqslant k_{2} \leqslant f\right)$ such that

(a) $\hat{r}_{1}-r_{1}<\cdots<\hat{r}_{k_{1}}-r_{k_{1}}$;

(b) $\hat{r}_{k_{1}}-r_{k_{1}}=\cdots=\hat{r}_{k_{2}}-r_{k_{2}}$;

(c) $\hat{r}_{k_{2}}-r_{k_{2}}>\cdots>\hat{r}_{f}-r_{f}$.

In (1), the rent differences form a convex shape, and in (2), they form a concave shape. If $k_{1}=$ $k_{2}=1$, the rent differences are just increasing in (1) and just decreasing in (2). If $k_{1}=k_{2}=f$, we have oppositely monotone sequences. In either (1) or (2), there may be both increasing and decreasing segments. The theorem also states that the segments of constant differences may be possible, but such segments are rare cases, since small perturbations of some parameters, e.g., incomes, destroy these segments, which will be discussed in detail in Section 6 .

Proof of Theorem 5.2. We show (2), and (1) can be proved in the dual manner. Let $k_{1}$ be the largest number among $k$ 's satisfying $\hat{r}_{1}-r_{1}<\cdots<\hat{r}_{k}-r_{k}$. If $\hat{r}_{1}-r_{1} \geqslant \hat{r}_{2}-r_{2}$, then $k_{1}=1$. Let $k_{2}$ be the largest number among $k$ 's satisfying $\hat{r}_{k_{1}}-r_{k_{1}}=\cdots=\hat{r}_{k}-r_{k}$. This $k_{2}$ may be $k_{1}$ or $f$. The definitions of $k_{1}$ and $k_{2}$ imply (a) and (b). By the choice of $k_{2}$, we have $\hat{r}_{k_{2}}-r_{k_{2}}>$ $\hat{r}_{k_{2}+1}-r_{k_{2}+1}$. In the former case, by Theorem 5.1(2), we have (c).

The cases (1) and (2) of Theorems 5.1 and 5.2 exhibit the dual structure with the opposite inequalities. It is the point that we have only these two types of changes.

Naive forms of the above theorems were found in Kaneko [15]. Specifically, Theorems 5.1(1) and 5.2(1) are generalizations of the corresponding results in a more restricted environment in [15]. However, the dual structures are not touched at all. 
The above two theorems can be used to have more concrete comparative statics. Here we give only one instance. The following is a comparative statics result when only the marginal cost for the marginal category goes up under the assumption that the other parameters are all fixed. The increase in the rent of the marginal category $f$ is interpreted as expressing the increase in costs for providing apartments in the marginal category $f$, that is, $r_{f}<\hat{r}_{f}$. For the interpretation of costs for apartments in the marginal category $f$, see the paragraph before Example 2.7.

Corollary 5.3. Let $(M, N)$ and $(\widehat{M}, \widehat{N})$ be two markets with $f=\hat{f}$ and $I_{G(k)}=\hat{I}_{\widehat{G}(k)}$ for $k=$ $1, \ldots, f-1$. Let $r$ and $\hat{r}$ be the differential rent vectors in $(M, N)$ and $(\widehat{M}, \widehat{N})$ with $r_{f}<\hat{r}_{f}$. Then $0<\hat{r}_{1}-r_{1}<\cdots<\hat{r}_{f}-r_{f}$.

Proof. By the rent equation (2.5), we have $u\left(\mathbf{e}^{f-1}, I_{G(f-1)}-r_{f-1}\right)=u\left(\mathbf{e}^{f}, I_{G(f-1)}-r_{f}\right)$. Let $\delta=\hat{r}_{f}-r_{f}$. Then $u\left(\mathbf{e}^{f-1}, I_{G(f-1)}-r_{f-1}-\delta\right)<u\left(\mathbf{e}^{f}, I_{G(f-1)}-r_{f}-\delta\right)=u\left(\mathbf{e}^{f}, I_{G(f-1)}-\hat{r}_{f}\right)$. Since $u\left(\mathbf{e}^{f-1}, I_{G(f-1)}-\hat{r}_{f-1}\right)=u\left(\mathbf{e}^{f}, I_{G(f-1)}-\hat{r}_{f}\right)$, we have, by Assumption A, $I_{G(f-1)}-$ $r_{f-1}-\delta<I_{G(f-1)}-\hat{r}_{f-1}$, i.e., $\hat{r}_{f-1}-r_{f-1}<\hat{r}_{f}-r_{f}$. By Theorem 5.1(2), we have $\hat{r}_{1}-r_{1}<\cdots<\hat{r}_{f}-r_{f}$. Also, since $u\left(\mathbf{e}^{f-1}, I_{G(f-1)}-\hat{r}_{f-1}\right)=u\left(\mathbf{e}^{f}, I_{G(f-1)}-\hat{r}_{f}\right)<u\left(\mathbf{e}^{f}\right.$, $\left.I_{G(f-1)}-r_{f}\right)=u\left(\mathrm{e}^{f-1}, I_{G(f-1)}-r_{f-1}\right)$, we have $0<\hat{r}_{f-1}-r_{f-1}$. In the same manner, we can prove $0<\hat{r}_{1}-r_{1}$.

Ito [12] considered a different type of comparative statics, i.e., the quality of apartments of one category changes. The above results (especially Theorem 4.1) are useful in such a study.

\section{The bending points in the rent differences}

We call the apartment categories $k_{1}$ and $k_{2}$ given in Theorem 5.2 the upper and lower bending points of rent differences. These points divide the rent differences into the increasing segment, constant segment, and decreasing segment. These points are useful in knowing the effects of parameter changes. In this section, we estimate their locations from some basic information. Also, we will show that the presence of the constant segment is a rare case, i.e., these two points coincide typically. In the following, we assume $f \leqslant \hat{f}$.

Theorem 5.2 states the existence of such bending points, but does not exclude the trivial cases such as $k_{1}=k_{2}=1$ or $k_{1}=k_{2}=f$. The following theorem restricts the range of the locations of the bending points $k_{1}$ and $k_{2}$ by using the rent difference of the marginal category $f$ and the boundary income difference of category $t$.

Theorem 6.1 (Conditions for Nontrivial Bending Points). Let $r$ and $\hat{r}$ be differential rent vectors in $(M, N)$ and $(\widehat{M}, \widehat{N})$. Let $t$ be any category $(1 \leqslant t<f)$.

(1) Under (5.1),

(a) if $\hat{I}_{\widehat{G}(t)}-I_{G(t)}>\hat{r}_{f}-r_{f}$, then there is a $k_{1}\left(t<k_{1} \leqslant f\right)$ such that $\hat{r}_{1}-r_{1}>\cdots>$ $\hat{r}_{k_{1}}-r_{k_{1}}$ and $\hat{r}_{k_{1}}-r_{k_{1}} \leqslant \cdots \leqslant \hat{r}_{f}-r_{f}$

(b) if $\hat{I}_{\widehat{G}(t)}-I_{G(t)}<\hat{r}_{f}-r_{f}$, then there is a $k(t \leqslant k<f)$ such that $\hat{r}_{k}-r_{k}<\cdots<\hat{r}_{f}-r_{f}$.

(2) Under (5.2),

(a) if $\hat{I}_{\widehat{G}(t)}-I_{G(t)}>\hat{r}_{f}-r_{f}$, then there is a $k(t \leqslant k<f)$ such that $\hat{r}_{k}-r_{k}>\cdots>\hat{r}_{f}-r_{f}$;

(b) if $\hat{I}_{\widehat{G}(t)}-I_{G(t)}<\hat{r}_{f}-r_{f}$, then there is a $k_{1}\left(t<k_{1} \leqslant f\right)$ such that $\hat{r}_{1}-r_{1}<\cdots<$ $\hat{r}_{k_{1}}-r_{k_{1}}$ and $\hat{r}_{k_{1}}-r_{k_{1}} \geqslant \cdots \geqslant \hat{r}_{f}-r_{f}$. 
Proof. We prove only (2). Consider (a). Suppose $\hat{I}_{\widehat{G}(t)}-I_{G(t)}>\hat{r}_{f}-r_{f}$. First, we show that

$$
\hat{I}_{\widehat{G}(k)}-I_{G(k)}>\hat{r}_{k}-r_{k} \text { for some } k \quad(t \leqslant k<f) .
$$

Now, we show the contrapositive that the negation of (6.1) implies $\hat{I}_{\widehat{G}(t)}-I_{G(t)} \leqslant \hat{r}_{f}-r_{f}$. Suppose that $\hat{I}_{\widehat{G}(k)}-I_{G(k)} \leqslant \hat{r}_{k}-r_{k}$ for all $k(t \leqslant k<f)$. Then, by Theorem 4.1, we have $\hat{r}_{k}-r_{k} \leqslant \hat{r}_{k+1}-r_{k+1}$ for all $k=t, \ldots, f-1$. This together with $\hat{I}_{\widehat{G}(t)}-I_{G(t)} \leqslant \hat{r}_{t}-r_{t}$ imply $\hat{I}_{\widehat{G}(t)}-I_{G(t)} \leqslant \hat{r}_{f}-r_{f}$. We have prove the contrapositive. Thus we have (6.1).

By (6.1) and Theorem 4.1, we have $\hat{r}_{k}-r_{k}>\hat{r}_{k+1}-r_{k+1}$. This together with Theorem 5.1(2) implies that $\hat{r}_{k}-r_{k}>\cdots>\hat{r}_{f}-r_{f}$.

Consider (b). Suppose $\hat{I}_{\widehat{G}(t)}-I_{G(t)}<\hat{r}_{f}-r_{f}$. It holds that

$$
\hat{I}_{\widehat{G}(k)}-I_{G(k)}<\hat{r}_{k}-r_{k} \text { for some } k \quad(t \leqslant k<f) .
$$

This can be proved in the same manner as (6.1). By (6.2) and Theorem 4.1, we have $\hat{r}_{k}-r_{k}<$ $\hat{r}_{k+1}-r_{k+1}$. This together with Theorem 5.1(2) implies that $\hat{r}_{1}-r_{1}<\cdots<\hat{r}_{k+1}-r_{k+1}$.

Let $k_{1}$ be the largest integer among such $(k+1)$ 's. Hence we have the latter inequalities of (a). By Theorem 5.2(2), we have $\hat{r}_{k_{1}}-r_{k_{1}} \geqslant \cdots \geqslant \hat{r}_{f}-r_{f}$.

Each claim of Theorem 6.1 has two statements. These are combined as follows: Let $k_{1}$ and $k_{2}$ be upper and lower bending points.

Corollary 6.2 (Locations of the Bending Points). Let $r$ and $\hat{r}$ be differential rent vectors in $(M, N)$ and $(\widehat{M}, \widehat{N})$. Let $t$ be a category number with $t<f-1$.

(1) Under (5.1), if $\hat{I}_{\widehat{G}(t)}-I_{G(t)}>\hat{r}_{f}-r_{f}>\hat{I}_{\widehat{G}(t+1)}-I_{G(t+1)}$, then $t+1 \leqslant k_{1} \leqslant k_{2}<f$.

(2) Under (5.2), if $I_{\widehat{G}(t)}-I_{G(t)}<\hat{r}_{f}-r_{f}<\hat{I}_{\widehat{G}(t+1)}-I_{G(t+1)}$, then $t+1 \leqslant k_{1} \leqslant k_{2}<f$.

Proof. Consider (2). Suppose $I_{\widehat{G}(t)}-I_{G(t)}<\hat{r}_{f}-r_{f}<\hat{I}_{\widehat{G}(t+1)}-I_{G(t+1)}$. Then, by Theorem 6.1(2), $t+1 \leqslant k_{1} \leqslant f$ and $t+1 \leqslant k_{2}<f$. By Theorem 5.2(2), we have $k_{1} \leqslant k_{2}$.

In Theorem 6.1 and Corollary 6.2, the locations of the upper and lower bending points are restricted, but they may still differ. Now we argue that the they typically coincide or differ by 1 .

The next theorem gives a condition for the coincidence of the upper and lower bending points.

Theorem 6.3 (Condition for a unique Bending Point). Let $r$ and $\hat{r}$ be differential rent vectors in $(M, N)$ and $(\widehat{M}, \widehat{N})$. Let $k_{1}, k_{2}$ be the upper and lower bending points, and $t$ a category number with $t<f-1$.

(1) Under (5.1), $\hat{I}_{\widehat{G}(t)}-I_{G(t)}>\hat{r}_{t}-r_{t}$ and $\hat{I}_{\widehat{G}(t+1)}-I_{G(t+1)}<\hat{r}_{t+1}-r_{t+1}$ if and only if $k_{1}=$ $k_{2}=t+1$.

(2) Under (5.2), $\hat{I}_{\widehat{G}(t)}-I_{G(t)}<\hat{r}_{t}-r_{t}$ and $\hat{I}_{\widehat{G}(t+1)}-I_{G(t+1)}>\hat{r}_{t+1}-r_{t+1}$ if and only if $k_{1}=$ $k_{2}=t+1$.

Proof. Consider (2). Suppose $\hat{I}_{\widehat{G}(t)}-I_{G(t)}<\hat{r}_{t}-r_{t}$ and $\hat{I}_{\widehat{G}(t+1)}-I_{G(t+1)}>\hat{r}_{t+1}-r_{t+1}$. Then it follows from Theorem 4.1 that $\hat{r}_{t}-r_{t}<\hat{r}_{t+1}-r_{t+1}>\hat{r}_{t+2}-r_{t+2}$. By Theorem 5.1, we have 
$\hat{r}_{1}-r_{1}<\cdots<\hat{r}_{t+1}-r_{t+1}$ and $\hat{r}_{t+1}-r_{t+1}>\cdots>\hat{r}_{f}-r_{f}$. Thus, the upper and lower bending points $k_{1}$ and $k_{2}$ coincide with $t+1$.

The converse can be proved by tracing the above proof back.

Even when the inequalities in (5.1) are all strict, the upper and lower bending points $k_{1}$ and $k_{2}$ may not coincide, but they may differ by at most one, which is stated in the following corollary. Let $k_{1}, k_{2}$ be the upper and lower bending points.

Corollary 6.4 (Small Difference of the Bending Points). If (5.1) or (5.2) holds with strict inequalities, then $k_{1}=k_{2}$ or $k_{1}+1=k_{2}$.

Proof. Consider the case where (5.1) holds with strict inequalities. Then, let $\hat{r}_{k_{1}}-r_{k_{1}}=$ $\hat{r}_{k_{1}+1}-r_{k_{1}+1}$. Then $\hat{I}_{\widehat{G}\left(k_{1}\right)}-I_{G\left(k_{1}\right)}=\hat{r}_{k_{1}}-r_{k_{1}}=\hat{r}_{k_{1}+1}-r_{k_{1}+1}>\hat{I}_{\widehat{G}\left(k_{1}+1\right)}-I_{G\left(k_{1}+1\right)}$, which, by Theorem 4.1, implies $\hat{r}_{k_{1}+1}-r_{k_{1}+1}>\hat{r}_{k_{1}+2}-r_{k_{1}+2}$. It follows from this and Theorem 5.1 that $\hat{r}_{t}-r_{t}>\hat{r}_{t+1}-r_{t+1}$ for all $t>k_{1}$. Thus, $k_{2}=k_{1}+1$.

When (5.1) or (5.2) holds with strict inequalities, the upper and lower bending points coincide or differ by 1 . Even the latter case can be regarded as rare. In practice, the upper and lower bending points coincide.

\section{Application to a housing market in Tokyo (2)}

\subsection{Estimation of the basic utility function}

Let us continue the application of our housing market model $(M, N)$ to a rental housing market in Tokyo in Section 3. We assume that all the elements of our model other than the utility function $U(t, s, c)$ are the same as those given in Section 3. In this section, we discuss the estimation method for the utility function $\widehat{U}(t, s, c)=-2.2 t+3.1 s+80 \sqrt{c}$ of (3.1) and mention briefly how we evaluate this estimation. Here, we emphasize that the linearity of the utility function over $t$ and $s$ is the result of this estimation. In a separate paper, we will give a full statistical and econometric study of this estimation.

Let a utility function be given as:

$$
U(t, s, c)=-\alpha t+\beta s+\gamma \sqrt{c},
$$

where $\alpha, \beta, \gamma$ are positive parameters. Then, the differential rent vector $\left(r_{1}, \ldots, r_{20}\right)$ is determined by the rent equation (2.5) with a given $r_{20}$, i.e.,

$$
\left.\begin{array}{c}
U(\cdot, \cdot, \cdot) \\
r_{20}
\end{array}\right\} \longmapsto\left(r_{1}, \ldots, r_{20}\right) .
$$

We measure the distance of $\left(r_{1}, \ldots, r_{20}\right)$ to the actual data of rents $\left\{p_{1 d}\right\}_{d=1}^{113}, \ldots,\left\{p_{20 d}\right\}_{d=1}^{689}$ observed in the Yahoo Real Estate, 2004, Dec.15 by the total sum of square variations over categories $1, \ldots, T=20$ :

$$
\sum_{k=1}^{20} \sum_{d}\left(p_{k d}-r_{k}\right)^{2} \text {. }
$$

We minimize this distance controlling $r_{20}, \alpha, \beta$ and $\gamma$. 
We can fix $\gamma=80$ without influencing (7.2). Giving small grids for $r_{20}, \alpha, \beta$, and using a computer, we make an exhaustive search for the minimization of $\sum_{k=1}^{20} \sum_{d}\left(p_{k d}-r_{k}\right)^{2}$. The computation result is the utility function $\widehat{U}(t, s, c)=-2.2 t+3.1 s+80 \sqrt{c}$ of (3.1) together with the rent vector given in Table 3.2 and depicted in Figure 3.2.

Now, let $\bar{p}_{1}, \ldots, \bar{p}_{20}$ be the average rents of $\left\{p_{1 d}\right\}_{d=1}^{113}, \ldots,\left\{p_{20 d}\right\}_{d=1}^{689}$, e.g., $\bar{p}_{1}=\sum_{d=1}^{113} p_{1 d}$. Those averages have the special status in statistics. They minimize the total square variations with no constraints. That is,

$$
\sum_{k=1}^{20} \sum_{d}\left(p_{k d}-p_{k}\right)^{2} \quad \text { is minimized at } \quad\left(p_{1}, \ldots, p_{20}\right)=\left(\bar{p}_{1}, \ldots, \bar{p}_{20}\right) \text {. }
$$

Thus, we have $\sum_{k=1}^{20} \sum_{d}\left(p_{k d}-\bar{p}_{k}\right)^{2} \leqslant \sum_{k=1}^{20} \sum_{d}\left(p_{k d}-r_{k}\right)^{2}$. The left-hand sum is the total square variations from the average rent vector $\left(\bar{p}_{1}, \ldots, \bar{p}_{20}\right)$, and the right-hand sum is that from the differential rent vector $\left(r_{1}, \ldots, r_{20}\right)$ we estimate. We adopt the left-hand sum as the point for criterion: We take the ratio of $\sum_{k=1}^{20} \sum_{d}\left(p_{k d}-r_{k}\right)^{2}$ to $\sum_{k=1}^{20} \sum_{d}\left(p_{k d}-\bar{p}_{k}\right)^{2}$. In our example, this ratio is given as

$$
\frac{\sum_{k=1}^{20} \sum_{d}\left(p_{k d}-r_{k}\right)^{2}}{\sum_{k=1}^{20} \sum_{d}\left(p_{k d}-\bar{p}_{k}\right)^{2}} \doteqdot \frac{1701510.4}{1525350.6} \doteqdot 1.1154 .
$$

Thus, our model can approximate the average rent vectors within the accuracy of (7.5).

In the above calculation, we have already assumed that the utility function $U(t, s, c)$ is of the form (7.1). However, the above calculation can be done in a different class of utility functions:

$$
U(t, s, c)=\sqrt{\alpha_{2}-\alpha_{1} t}+\sqrt{\beta_{1} s+\beta_{2}}+\gamma \sqrt{c} .
$$

This satisfies the law of diminishing marginal utility for each variable, while (7.1) does not satisfy it for $t$ and $s$ in a strict sense. In this sense, the utility function of (7.6) is more compatible with the standard textbook teaching than the utility function $U(t, s, c)$ of (7.1). However, by the computer experiments, we can show that $\sum_{k=1}^{20} \sum_{d}\left(p_{k d}-r_{k}\right)^{2}$ is getting smaller as $\alpha_{2}$ and $\beta_{2}$ become larger in (7.6). This can be interpreted as meaning that we have a better estimation result when $U(t, s, c)$ become close to linear in variables $t$ and $s$. Also, it is a result of computation that the total sum of square variations corresponding to (7.1) always dominates that corresponding to (7.6). Thus, the estimated utility function $\widehat{U}(t, s, c)$ is linear for $t$ and $s$. In fact, the linearity for the consumption $c$ is not obtained, i.e., the law of diminishing marginal utility holds only for consumption.

We will discuss the details of the above statistical and econometric study in a separate paper.

\subsection{Comparative statics in the rental housing market along the Chuo-line}

Let us continue the rental housing market in Section 3. Now, suppose that the boundary incomes in the market $(M, N)$ increase by $1 \%$ uniformly, and the rent $r_{20}$ for a unit of category 20 increases by $10 \%$. We use the estimated utility function $\widehat{U}(t, s, c)=-2.2 t+3.1 s+80 \sqrt{c}$ of (3.1) for $(M, N)$, but we assume the same utility function for $(\widehat{M}, \widehat{N})$. The other parameters are assumed to be the same. ${ }^{12}$ Here, we assume to know $\hat{r}_{20}=1.1 \times r_{20}$.

\footnotetext{
12 It may be asymmetric to have 1 and $10 \%$ increments, respectively, in the incomes and valuation $a_{20}=p_{20}$. However, the valuation $a_{20}$ reflects the cost of building an apartment, and a change in the valuation may be different from those in the incomes of households.
} 
In this case, condition (5.1) holds, i.e., $I_{\widehat{G}(1)}-I_{G(1)}>\cdots>I_{\widehat{G}(19)}-I_{G(19)}$. Also,

$$
I_{\widehat{G}(1)}-I_{G(1)}=8.9>\hat{r}_{20}-r_{20}=4.96 .
$$

Thus the income difference for the boundary household $G(1)$ is 8900 yen and is greater than the rent difference 4960 yen. Theorem 6.1 implies that the bending point $k_{1}>1$ exists. In fact, we can get better information from Table 7.1: we find

$$
I_{\widehat{G}(14)}-I_{G(14)}=5.48>\hat{r}_{20}-r_{20}=4.96>3.93=I_{\widehat{G}(15)}-I_{G(15)} .
$$

Then, we can use Corollary 6.2 to obtain $15 \leqslant k_{1} \leqslant k_{2}<20$. Even Theorem 6.3 gives the precise answer $k_{1}=k_{2}=15$, since $\hat{r}_{14}-r_{14}=4.804<I_{\widehat{G}(14)}-I_{G(14)}$ and $I_{\widehat{G}(15)}-I_{G(15)}<\hat{r}_{15}-r_{15}=$ 4.800. Then, we can estimate the shape of the differential rent curves (see Fig. 7.1).

Finally, we show that the remark stated in Section 5.1 about more one segments of increasing and decreasing segments of boundary income differences. More concretely, we gives a comparative statics with two segments of boundary income differentials. Suppose that the number of households increase from $\sum_{k=1}^{20} w_{k}=6194$ to $6194+100=6294$, and specifically that the supplies $w_{1}, \ldots, w_{5}$ increase by 20 . That is, $\widehat{w}_{1}=133, \widehat{w}_{2}=83, \widehat{w}_{3}=40, \widehat{w}_{4}=50, \widehat{w}_{5}=270$, but

\begin{tabular}{|c|c|c|c|c|c|c|}
\hline \multirow[b]{2}{*}{$k$} & \multicolumn{3}{|c|}{ Changes in $I_{G(k)}(1000$ yen $)$} & \multicolumn{3}{|c|}{ Changes in rents (1000 yen) } \\
\hline & $I_{G(k)}$ & $\hat{I}_{\widehat{G}}(k)$ & $\hat{I}_{\widehat{G}(k)}-I_{G(k)}$ & $r_{k}$ & $\hat{r}_{k}$ & $\hat{r}_{k}-r_{k}$ \\
\hline 1 & 885.53 & 894.39 & 8.86 & 194.19 & 199.25 & 5.05 \\
\hline 2 & 877.39 & 886.17 & 8.77 & 179.66 & 184.67 & 5.01 \\
\hline : & : & : & : & : & : & : \\
\hline 14 & 548.25 & 553.73 & 5.48 & 83.22 & 88.02 & 4.804 \\
\hline 15 & 392.72 & 396.64 & 3.93 & 77.05 & 81.85 & 4.800 \\
\hline$:$ & $:$ & : & : & : & : & : \\
\hline 20 & 100.00 & 101.00 & 1.00 & 49.59 & 54.55 & 4.96 \\
\hline
\end{tabular}

Table 7.1

\begin{tabular}{|c|c|c|c|c|c|c|}
\hline \multirow[b]{2}{*}{$k$} & \multicolumn{3}{|c|}{ Changes in $I_{G(k)}(1000$ yen $)$} & \multicolumn{3}{|c|}{ Changes in rents (1000 yen) } \\
\hline & $I_{G(k)}$ & $\hat{I}_{\widehat{G}(k)}$ & $\hat{I}_{\widehat{G}(k)}-I_{G(k)}$ & $r_{k}$ & $\hat{r}_{k}$ & $\hat{r}_{k}-r_{k}$ \\
\hline 1 & 885.53 & 883.22 & -2.31 & 194.195 & 189.088 & -5.107 \\
\hline 2 & 877.39 & 872.67 & -4.73 & 179.658 & 174.522 & -5.136 \\
\hline 3 & 874.81 & 867.58 & -7.23 & 169.451 & 164.312 & -5.139 \\
\hline 4 & 870.93 & 861.23 & -9.71 & 160.660 & 155.534 & -5.126 \\
\hline 5 & 838.64 & 826.90 & -11.74 & 153.044 & 147.942 & -5.102 \\
\hline 6 & 834.77 & 823.09 & -11.68 & 143.190 & 138.136 & -5.054 \\
\hline 7 & 809.32 & 798.05 & -11.27 & 138.580 & 133.548 & -5.032 \\
\hline$\vdots$ & $\vdots$ & $\vdots$ & $\vdots$ & $\vdots$ & $\vdots$ & $\vdots$ \\
\hline 14 & 548.25 & 541.13 & -7.12 & 83.218 & 78.405 & -4.813 \\
\hline 15 & 392.72 & 388.07 & -4.65 & 77.052 & 72.254 & -4.798 \\
\hline$\vdots$ & $\vdots$ & $\vdots$ & $\vdots$ & $\vdots$ & $\vdots$ & $\vdots$ \\
\hline 20 & 100.00 & 100.00 & 0.00 & 49.587 & 44.628 & -4.959 \\
\hline
\end{tabular}

Table 7.2 


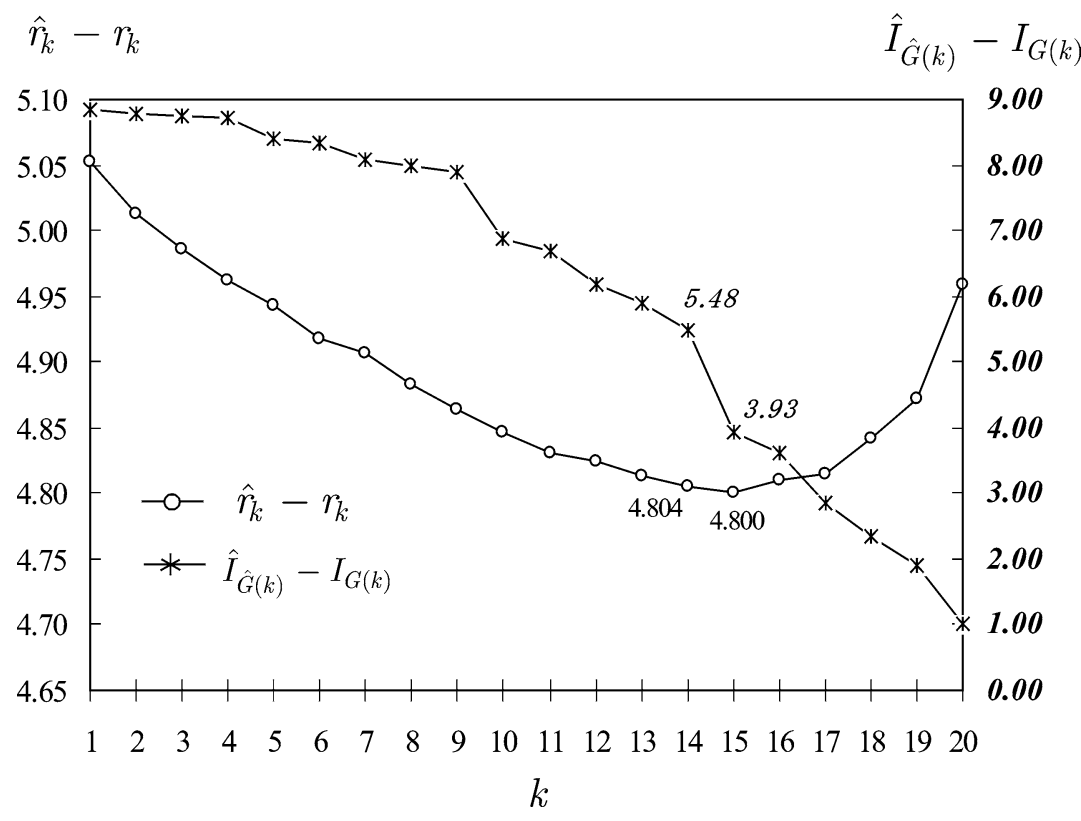

Fig. 7.1. $k_{1}=k_{2}=15$.

$k$

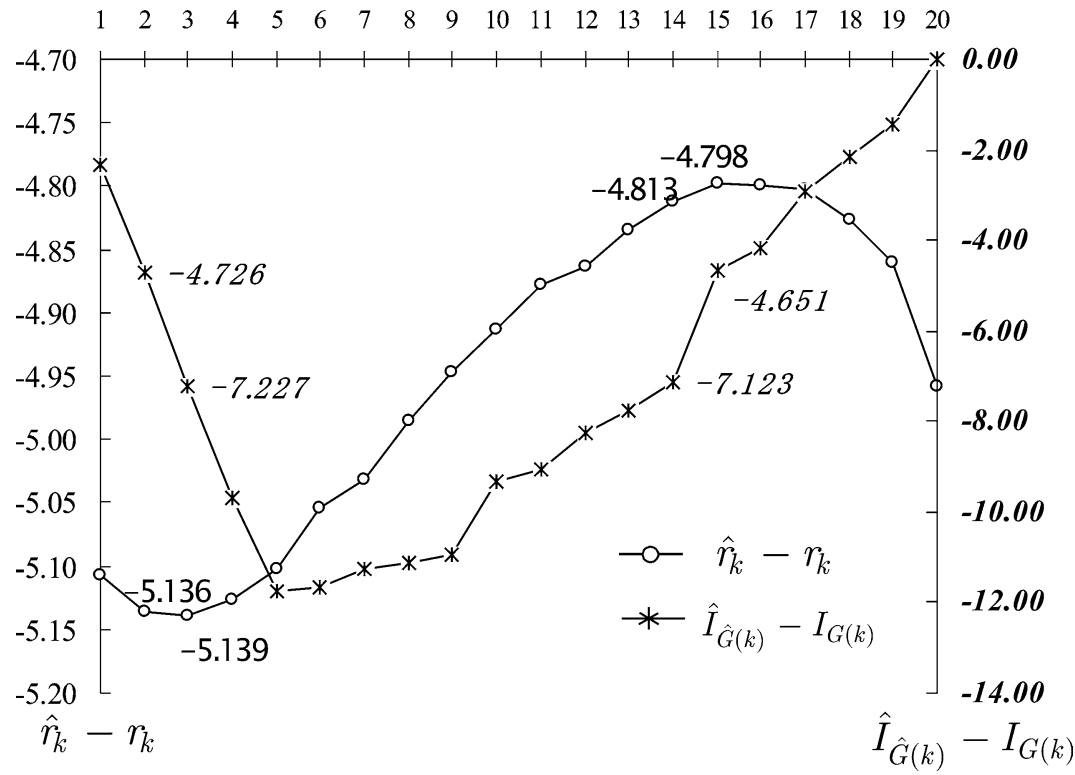

Fig. 7.2. Bending points in the two segments.

we assume $\widehat{w}_{k}=w_{k}$ for $k=6, \ldots, 20$. Also the incomes are still distributed uniformly from 100 to 900 thousand yen. With above changes, it holds that

$$
I_{\widehat{G}(1)}-I_{G(1)}>\cdots>I_{\widehat{G}(5)}-I_{G(5)} \quad \text { and } \quad I_{\widehat{G}(6)}-I_{G(6)}<\cdots<I_{\widehat{G}(19)}-I_{G(19)} .
$$


Thus, we have the two segments of income differentials, which are calculated in Table 7.2.

One more change is that $r_{20}$ decreases by $10 \%$. Then, we can calculate the new differential rent vector, which is also given in Table 7.2. Since the income differential lines are divided into the two parts from 1 to 5 and from 6 to 20, it follows from Theorem 6.3 that each segment has one bending point, i.e., the upper and lower bending points coincide with 3 in the left segment, and they coincide with 15 in the right segment. These are depicted in Fig. 7.2.

\section{Concluding remarks}

We give three remarks on future possible developments of our study of rental housing markets. One is more on comparative statics, the second is on econometrics, and the third is on the possibility of relaxing the assumption of an identical utility function.

We have focussed on the theoretical sides of comparative statics, particularly, on the dual relationships between the rents for categories and the boundary income differences. We have not looked at direct cause-effect relationships between basic parameters (mentioned in Section 4) and rents. Ito [12] gave a direct cause-effect comparative statics along the line of our research. There could be many possible comparative statistical studies on such relationships. For these, computer simulations may play more roles than theoretical studies, since we have many cases of comparisons. We have been developing computer simulation methods for such comparisons, which is still an ongoing project.

For further comparative statics as well as more practical studies, it would be useful to develop an econometric method so as to have an estimation of the utility function such as $\widehat{U}(s, t, c)=-2.2 t+3.1 s+80 \sqrt{c}$ of (3.1). The present authors have been practicing such an estimation method. As mentioned in Section 7.1, this utility function is estimated by minimizing, over possible coefficients for the utility function, the total sum of square variations. So far, estimation works well, and some interesting findings have been revealed, for example, the utility function does not strictly satisfy the law of diminishing marginal utilities over the leisure time as well as the housing size, but does in consumption. This will be discussed in a separate paper.

The final remark is about the possibility of eliminating Assumption C (Identical Utility Function). This assumption is interpreted as restricting our scope to a housing market with one office district. If a housing market has two or more office districts to which households commute, then Assumption $\mathrm{C}$ should be relaxed so that only the households who commute to the same business district have identical utility functions.

More concretely, the set of consumers $M$ is partitioned into $M_{1}, \ldots, M_{D}$ : each household $i$ in $M_{k}(k=1, \ldots, D)$ has the same utility function $u_{k}:\left\{\mathbf{e}^{0}, \mathbf{e}^{1}, \ldots, \mathbf{e}^{T}\right\} \times R_{+} \rightarrow R$. In an application, this utility function is regarded as derived from a larger utility function $U(t, s, c)$ as in Section 3. Here, each household $i \in M_{k}$ has his office in some location and $t$ is the time-distance from his office to an apartment. Variables $s$ and $c$ are not affected by its location. For example, the Sobu JR line connects Tokyo station to Chiba station, and some people have offices in Chiba. Then, the households are divided into two groups: the ones commuting to Tokyo station and the ones commuting to Chiba. Then the time-distance $t$ to the office location is depending upon a household. In this formulation, we have two derived utility functions $u_{T}$ for households to Tokyo station and $u_{C}$ for ones to Chiba station.

To study the housing market with multiple business districts, both theoretical and simulation analyses are required. For example, some general results such as the existences of an equilibrium and of a maximal rent vector (Theorems 2.1 and 2.2) remain. However, the Rent Equation Theorem (Theorem 4.1) is no longer available at least in the present form. In this case, Miyake's [17] 
graph-theoretic consideration of the maximal rent vector may help. Since the fixed-point algorithm is available in the general case (see Kaneko-Yamamoto [13]), we may develop a computer simulation analysis.

Our results of comparative statics could be extended to such housing markets with multiple business districts, where Assumption E (Normality) is needed. If we find a segment of households who have the same utility function and are ordered with income differences such as (5.1) and/or (5.2), we would obtain the comparative statics results, restricting our attention to such a segment. Nevertheless, we should know how such segments occur with other segments. This is a theoretical problem for comparative statics in housing markets with multiple business districts.

\section{Acknowledgments}

The authors are grateful for numerous kind comments by the Editor, Jan K. Brueckner, and two referees of this journal.

\section{Appendix A. Proof of Theorem 2.6 in case (2)}

To prove Theorem 2.6 in case (2), we need one lemma.

Lemma A. Let $(p, x, y)$ be a maximal competitive equilibrium. For each category $k=1, \ldots$, $f-1$, there are households $i, i^{\prime} \in M$ such that $x_{i}=\mathbf{e}^{k}, I_{i}=I_{G(k)}$ and $x_{i^{\prime}}=\mathbf{e}^{k+1}, I_{i^{\prime}}=I_{G(k)+1}$.

Proof. We show the existence of $i$. Suppose, on the contrary, that there is no $i \in M$ such that $x_{i}=\mathrm{e}^{k}$ and $I_{i}=I_{G(k)}$. There are two cases to be considered, and either case is shown to be impossible. First, there is an $i$ such that $I_{i}=I_{G(k)}, x_{i}=\mathbf{e}^{l}$ and $l>k$. Then, by the supposition and Lemma 2.3(2), it holds that $I_{i^{\prime}}>I_{G(k)}$ for all $i^{\prime} \in M$ with $x_{i^{\prime}}=\mathbf{e}^{t}(t \leqslant k)$. However, this is impossible by the definition of $G(k)$. Second, there is an $i$ such that $I_{i}=I_{G(k)}, x_{i}=\mathbf{e}^{l}$ and $k>l$. By the supposition and Lemma 2.3(2), it holds that $I_{i^{\prime}}<I_{G(k)}$ for all $i^{\prime} \in M$ with $x_{i^{\prime}}=\mathbf{e}^{t}$ $(t \geqslant k)$. This is also impossible.

Proof of case (2) of Theorem 2.6. Now, suppose, on the contrary, that (2.5) fails. Then there is a category $t(1 \leqslant t \leqslant f-1)$ such that

$$
\begin{aligned}
& u\left(\mathbf{e}^{k}, I_{G(k)}-p_{k}\right)=u\left(\mathbf{e}^{k+1}, I_{G(k)}-p_{k+1}\right) \text { for } k=1, \ldots, t-1 \\
& u\left(\mathbf{e}^{t}, I_{G(t)}-p_{t}\right)>u\left(\mathbf{e}^{t+1}, I_{G(t)}-p_{t+1}\right) .
\end{aligned}
$$

Then we increase $p_{t}, p_{t-1}, \ldots, p_{1}$ slightly into $p_{t}^{\prime}, p_{t-1}^{\prime}, \ldots, p_{1}^{\prime}$ so that

(A1) $p_{k}^{\prime}<C_{k}\left(y_{k}+1\right)-C_{k}\left(y_{k}\right)$ for $k=1, \ldots, t-1$

(A2) $u\left(\mathbf{e}^{k}, I_{G(k)}-p_{k}^{\prime}\right)=u\left(\mathbf{e}^{k+1}, I_{G(k)}-p_{k}^{\prime}\right)$ for $k=1, \ldots, t-1$, and $u\left(\mathbf{e}^{t}, I_{G(t)}-p_{t}^{\prime}\right)>$ $u\left(\mathbf{e}^{t+1}, I_{G(t)}-p_{t+1}\right)$.

We can find these $p_{t}^{\prime}, p_{t-1}^{\prime}, \ldots, p_{1}^{\prime}$ satisfying $A 1$ and $A 2$. Indeed, observe that for any $k(0 \leqslant$ $k \leqslant f-1)$, since $u\left(\mathbf{e}^{k}, I_{i}-p_{k}\right) \geqslant u\left(\mathbf{0}, I_{i}\right)>u\left(\mathbf{e}^{k}, 0\right)$ by utility maximization at $\left(\mathbf{e}^{k}, I_{i}-p_{k}\right)$ and Assumption A, we have $I_{i}-p_{k}>0$. Hence we can increase $r_{t}$ slightly to $p_{t}^{\prime}$ so that $p_{t-1}^{\prime}, \ldots, p_{1}^{\prime}$ satisfy satisfying $A 1$ and $A 2$. 
Now, we define the rent vector $p^{*}$ by $p_{k}^{*}=p_{k}^{\prime}$ if $k \leqslant t$ and $p_{k}^{*}=p_{k}$ if $k>t$. Once we prove that $\left(p^{*}, x, y\right)$ is a competitive equilibrium, we would have a contradiction since $p$ is a maximal competitive rent vector. The balance of total demands and supplies holds for $(x, y)$. Since $p_{1}^{\prime}, \ldots, p_{t-1}^{\prime}$ are increased but satisfy $A 1$, profit maximization holds. It remains to show utility maximization for each household $i$. Utility maximization is almost directly derived for each household $i$ with $x_{i}=\mathbf{e}^{k}$ and $k>t$, since the rent he is facing is unchanged but the other rents are increased or unchanged. Now, consider a household $x_{i}=\mathbf{e}^{k}$ and $k \leqslant t$. It follows from Assumption $\mathrm{E}$ that for any $k^{\prime}<k$ and any $k^{\prime \prime}>k$, we have

$$
u\left(\mathbf{e}^{k^{\prime}}, I_{i}-p_{k^{\prime}}^{*}\right) \leqslant u\left(\mathbf{e}^{k^{\prime}+1}, I_{i}-p_{k^{\prime}+1}^{*}\right) \quad \text { and } u\left(\mathbf{e}^{k^{\prime \prime}}, I_{i}-p_{k^{\prime \prime}}^{*}\right) \geqslant u\left(\mathbf{e}^{k^{\prime \prime}+1}, I_{i}-p_{k^{\prime \prime}+1}^{*}\right) .
$$

Combining these, $u\left(\mathbf{e}^{k}, I_{i}-p_{k}^{*}\right) \geqslant u\left(\mathbf{e}^{l}, I_{i}-p_{l}^{*}\right)$ for all $l$.

\section{References}

[1] W. Alonso, Location and Land Use, Harvard Univ. Press, Cambridge, 1964.

[2] R.J. Arnott, R.M. Braid, A filtering model with steady-state housing, Regional Science and Urban Economics 27 (1997) 515-546.

[3] R.J. Arnott, R. Davidson, D. Pines, Spatial aspects of housing quality, density, and maintenance, Journal of Urban Economics 19 (1986) 190-217.

[4] R.J. Arnott, J.G. MacKinnon, W.C. Wheaton, Note: The welfare implications of spatial interdependence: An extension of Wheaton's “Optimal Distribution of Income Among Cities," Journal of Urban Economics 5 (1978) $131-136$.

[5] R.M. Braid, The short-run comparative statics of a rental housing market, Journal of Urban Economics 10 (1981) 286-310.

[6] R.M. Braid, The effects of government housing policies in a vintage filtering model, Journal of Urban Economics 16 (1984) 272-296.

[7] M. Fujita, Urban Economic Theory: Land Use and City Size, Cambridge Univ. Press, Cambridge, 1989.

[8] D. Gale, L.S. Shapley, College admissions and the stability of marriage, American Mathematical Monthly 69 (1962) 9-15.

[9] R.I. Gerber, Existence and description of housing market equilibrium, Regional Science and Urban Economics 15 (1985) 383-401.

[10] J. Hartwick, U. Schweizer, P. Varaiya, Comparative statics of a residential economy with several classes, Journal of Economic Theory 13 (1976) 396-413.

[11] D.R. Haurin, The effect of reimbursement of worker's transport costs: The case of urban areas in Japan, Journal of Urban Economics 13 (1983) 205-215.

[12] T. Ito, Effects of quality changes in rental housing markets, Mimeo, 2004.

[13] M. Kaneko, Y. Yamamoto, The existence and computation of competitive equilibria in markets with an indivisible commodity, Journal of Economic Theory 38 (1986) 118-136.

[14] M. Kaneko, The central assignment game and the assignment markets, Journal of Mathematical Economics 10 (1982) 205-232.

[15] M. Kaneko, Housing market with indivisibilities, Journal of Urban Economics 13 (1983) 22-50.

[16] G. van der Laan, D. Talman, Z. Yang, Existence and welfare properties of equilibrium in an exchange economies with multiple divisible and indivisible commodities and linear production, Journal of Economic Theory 103 (2002) 411-428.

[17] M. Miyake, Comparative statics of assignment markets with general utilities, Journal of Mathematical Economics 23 (1994) 519-531.

[18] R.F. Muth, Cities and Housing, Univ. of Chicago Press, 1969.

[19] D. Ricardo, The Principles of Political Economy and Taxation (Original work published 1817), J.M. Dent and Sons, London, 1965.

[20] J.C. Ohls, Public policy toward low income housing and filtering in housing markets, Journal of Urban Economics 2 (1975) 144-171.

[21] L.S. Shapley, M. Shubik, Assignment game I: The core, International Journal of Game Theory 1 (1972) 111-130.

[22] J.L. Sweeney, Quality, commodity hierarchies, and housing markets, Econometrica 42 (1974) 147-168.

[23] J.L. Sweeney, A commodity hierarchy model of the rental housing market, Journal of Urban Economics 1 (1974) 288-323. 
[24] J. van Lierop, House price structure and market equilibrium, Journal of Urban Economics 11 (1982) 272-289.

[25] E. von Böhm-Bawerk, Positive Theory of Capital (W. Smart, Trans.) (Original work published 1891), Books for Libraries, New York, 1921.

[26] J. von Neumann, O. Morgenstern, Theory of Games and Economic Behavior, Princeton Univ. Press, Princeton, 1944.

[27] W.C. Wheaton, On the optimal distribution of income among cities, Journal of Urban Economics 3 (1976) 31-44.

[28] M.J. White, Location choice and commuting behavior in cities with decentralized employment, Journal of Urban Economics 24 (1988) 129-152. 